Pacific

Journal of

Mathematics

\title{
A RELATIVE TRACE FORMULA FOR PGL(2) IN THE LOCAL SETTING
}

BROOKE FEIGON 


\title{
A RELATIVE TRACE FORMULA FOR PGL(2) IN THE LOCAL SETTING
}

\author{
BROOKE FEIGON
}

In memory of Jonathan Rogawski

\begin{abstract}
We develop the local Kuznetsov trace formula on a unitary group in two variables for an unramified quadratic extension of local, non-Archimedean fields $E / F$ and compare it to a local relative trace formula on $\operatorname{PGL}(2, E)$. To define the local distributions for the relative trace formula, we define a regularized local period integral and prove that it is a $\operatorname{PGL}(2, F)$-invariant linear functional. By comparison of the two local trace formulas, we get an equality between a local PGL $(2, F)$-period and local Whittaker functionals.
\end{abstract}

1. Introduction 395

2. Notation 403

3. The local Kuznetsov trace formula for U(2) 405

4. The local relative trace formula and periods for PGL(2) 413

5. Comparison of local trace formulas and applications 427

Acknowledgments $\quad 430$

$\begin{array}{ll}\text { References } & 431\end{array}$

\section{Introduction}

Base change is an important type of functoriality which is useful in the study of automorphic forms by relating automorphic representations on different groups. Hervé Jacquet shed light on a new technique for attacking certain cases of Robert Langlands' important functoriality conjectures by comparing the relative and Kuznetsov trace formulas in the global setting. Jacquet's comparison of trace formulas leads to global identities that characterize the image of the base change map associating automorphic representations of a unitary group for a quadratic extension of number fields $E / F$ to automorphic representations of $\operatorname{GL}\left(2, \mathbb{A}_{E}\right)$ in terms of distinguished representations. While Jacquet's global identities factor, they do not give unique local identities.

The author was supported by NSF grant DMS-1201446.

MSC2010: 11F70, 22E50.

Keywords: relative trace formula. 
This paper uses techniques of James Arthur to define and develop a local Kuznetsov trace formula on U(2) and a local relative trace formula on GL(2). Both local trace formulas are expanded geometrically in terms of orbital integrals and spectrally in terms of local Bessel distributions and local relative Bessel distributions. The latter involve regularized local period integrals. We then carry out Jacquet's comparison in the local setting by relating these two local trace formulas for matching functions. This comparison yields identities between local Bessel distributions for automorphic representations on $\mathrm{U}(2)$ and local relative Bessel distributions for automorphic representations on GL(2).

Before we describe more precisely the local relative trace formula developed in this paper, let us recall the relative trace formula for GL(2). Take $E / F$ to be a quadratic extension of number fields and $\mathbb{A}_{F}$ to be the adeles of $F$. Let $\psi^{\prime}$ be a character on $F \backslash \mathbb{A}_{F} \cong N(F) \backslash N\left(\mathbb{A}_{F}\right)$ where $N$ is the upper triangular unipotent matrices of GL(2). Let $\psi=\psi^{\prime} \circ \operatorname{tr}_{E / F}$.

A cuspidal automorphic representation $\pi$ of $\operatorname{GL}\left(2, \mathbb{A}_{E}\right)$ with central character trivial on $\operatorname{GL}\left(2, \mathbb{A}_{F}\right)$ is distinguished by $\operatorname{GL}\left(2, \mathbb{A}_{F}\right)$ if there exists a $\phi \in V_{\pi}$, the vector space associated to $\pi$, such that the period integral, $P(\phi)$, is nonzero:

$$
P(\phi):=\int_{\mathrm{GL}(2, F) Z\left(\mathbb{A}_{F}\right) \backslash \mathrm{GL}\left(2, \mathbb{A}_{F}\right)} \phi(h) d h \neq 0 .
$$

Where $\pi^{\prime}$ is a cuspidal automorphic representation of the quasisplit unitary group $U\left(2, \mathbb{A}_{F}\right)$ and $\phi^{\prime} \in V_{\pi^{\prime}}$, let

$$
W\left(\phi^{\prime}\right)=\int_{N(F) \backslash N\left(\mathbb{A}_{F}\right)} \phi^{\prime}(n) \overline{\psi^{\prime}(n)} d n \quad \text { and } \quad W(\phi)=\int_{N(E) \backslash N\left(\mathbb{A}_{E}\right)} \phi(n) \overline{\psi(n)} d n .
$$

We define the Bessel distribution as

$$
B_{\pi^{\prime}}^{\prime}\left(f^{\prime}\right):=\sum_{i} W^{\prime}\left(\pi^{\prime}\left(f^{\prime}\right) \phi_{i}^{\prime}\right) \overline{W^{\prime}\left(\phi_{i}^{\prime}\right)}
$$

and the relative Bessel distribution as

$$
B_{\pi}(f):=\sum_{j} P\left(\pi(f) \phi_{j}\right) \overline{W\left(\phi_{j}\right)},
$$

where the summations are over an orthonormal basis of $V_{\pi^{\prime}}$ and $V_{\pi}$ respectively. Flicker [1991], following related work of Jacquet and Lai [1985] and Ye [1989], showed that for "matching functions" $f^{\prime}$ on $U\left(2, \mathbb{A}_{F}\right)$ and $f$ on $\operatorname{GL}\left(2, \mathbb{A}_{E}\right)$, if $\pi^{\prime}$ maps to $\pi$ under the unstable base change, then

$$
\sum_{i} W^{\prime}\left(\pi^{\prime}\left(f^{\prime}\right) \phi_{i}^{\prime}\right) \overline{W^{\prime}\left(\phi_{i}^{\prime}\right)}=\sum_{j} P\left(\pi(f) \phi_{j}\right) \overline{W\left(\phi_{j}\right)} .
$$

In particular, this equality characterizes the image of the unstable base change lift associating every automorphic representation of $U\left(2, \mathbb{A}_{F}\right)$ to an automorphic 
representation of $\operatorname{GL}\left(2, \mathbb{A}_{E}\right)$ in terms of $\operatorname{GL}\left(2, \mathbb{A}_{F}\right)$ distinguished representations. The equality above is proved via the relative trace formula [Jacquet 2005], which tells us that for $f$ and $f^{\prime}$ matching functions we have

$$
\begin{aligned}
\int_{\left(N(F) \backslash N\left(\mathbb{A}_{F}\right)\right)^{2}} K_{f^{\prime}}\left(n_{1}, n_{2}\right) & \psi^{\prime}\left(n_{1}^{-1} n_{2}\right) d n_{1} d n_{2} \\
= & \int_{\mathrm{GL}(2, F) Z\left(\mathbb{A}_{F}\right) \backslash \mathrm{GL}\left(2, \mathbb{A}_{F}\right)} \int_{N(E) \backslash N\left(\mathbb{A}_{E}\right)} K_{f}(h, n) \psi(n) d n d h
\end{aligned}
$$

where

$$
K_{f}(x, y)=\sum_{\delta \in Z(E) \backslash \mathrm{GL}(2, E)} f\left(x^{-1} \delta y\right) .
$$

The distributions $B_{\pi^{\prime}}^{\prime}\left(f^{\prime}\right)$ and $B_{\pi}(f)$ occur in the spectral expansions of the respective trace formulas.

In a different direction, Arthur [1989; 1991] developed a local version of the classical Arthur-Selberg trace formula. Let $G$ be a connected reductive algebraic group over a local field $F$ of characteristic zero. Diagonally embed $G(F)$ into $G(F) \times G(F)$. Then $L^{2}(G(F))$ is isomorphic to $L^{2}(G(F) \backslash G(F) \times G(F))$ by

$$
\phi \mapsto\left(\left(y_{1}, y_{2}\right) \mapsto \phi\left(y_{1}^{-1} y_{2}\right)\right) .
$$

For $\phi \in L^{2}(G(F))$, let $\left(\rho\left(g_{1}, g_{2}\right) \phi\right)(x)=\phi\left(g_{1}^{-1} x g_{2}\right)$. The right regular representation of $G(F) \times G(F)$ on $L^{2}(G(F) \backslash G(F) \times G(F)$ ) is equivalent to $\rho$ of $G(F) \times G(F)$ on $L^{2}(G(F))$. Thus to develop the local trace formula we look at $\rho(f)$ where $f=f_{1} \otimes f_{2} \in C_{c}^{\infty}(G(F) \times G(F))$. Then

$$
(\rho(f) \phi)(x)=\int_{G(F)} \int_{G(F)} f_{1}(g) f_{2}(y) \phi\left(g^{-1} x y\right) d g d y
$$

is an integral operator on $L^{2}(G(F))$ with kernel

$$
K_{f}(x, y)=\int_{G(F)} f_{1}(g) f_{2}\left(x^{-1} g y\right) d g .
$$

The local trace formula develops an explicit formula for the regularized trace of $\rho(f)$.

The main result of this paper is that, when evaluated with matching functions, the two local trace formulas described in Theorems 1.3 and 1.4 below, that is the local Kuznetsov trace formula and the local relative trace formula, are equal. Thus there is an equality between their local distributions on the spectral sides. This equality is stated in Theorem 1.1. This is the natural local counterpart to the global comparison from (1-1). In order to develop the local relative trace formula stated in Theorem 1.4, we have to define a local regularized period integral, prove it is a $\operatorname{GL}(2, F) \times \operatorname{GL}(2, F)$-invariant linear functional and relate it to the truncated 
period integral that initially appears in the relative trace formula. We state these properties about the local regularized period integral in Proposition 1.2.

To describe our results more precisely we need to introduce some further notation. Let $E / F$ now denote an unramified extension of local non-Archimedean fields of characteristic 0 . Let $O_{F}$ (respectively $O_{E}$ ) denote the ring of integers in $F$ (respectively $E$ ). Let $H=\mathrm{GL}(2) / F, G=\operatorname{Res}_{E / F} H$ and let

$$
G^{\prime}=\mathrm{U}(2, F)=\left\{g \in G:^{t} \bar{g}\left(\begin{array}{cc}
0 & 1 \\
-1 & 0
\end{array}\right) g=\left(\begin{array}{cc}
0 & 1 \\
-1 & 0
\end{array}\right)\right\} .
$$

Let $N^{\prime}$ and $N$ be the upper triangular unipotent matrices of $G^{\prime}$ and $G$, respectively, and let $M^{\prime}$ and $M$ be the diagonal subgroup of $G^{\prime}$ and $G$, respectively. Let $Z$ and $Z^{\prime}$ denote the center of $G$ and $G^{\prime}$, respectively. For any subgroup $X$ of $G$ let $\tilde{X}=Z \cap X \backslash X$ and let $X_{H}=X \cap H$. Let $\psi^{\prime}$ be an additive character on $F$ with conductor $\mathscr{O}_{F}$ and let $\psi(x)=\psi^{\prime} \circ \operatorname{tr}_{E / F}$. Let $f=f_{1} \otimes f_{2} \in C_{c}^{\infty}(\widetilde{G}(F) \times \widetilde{G}(F))$ and $f^{\prime}=f_{1}^{\prime} \otimes f_{2}^{\prime} \in C_{c}^{\infty}\left(\widetilde{G}^{\prime}(F) \times \widetilde{G}^{\prime}(F)\right)$.

We define the local Kuznetsov trace formula as the equality between the geometric expansion (in terms of orbital integrals) and spectral expansion (in terms of representations) of

$$
\lim _{t \rightarrow \infty} \int_{\left(N^{\prime} \times N^{\prime}\right)(F)} K_{f^{\prime}}\left(n_{1}, n_{2}\right) \psi^{\prime}\left(n_{1}^{-1} n_{2}\right) u\left(n_{1}, t\right) u\left(n_{2}, t\right) d n_{1} d n_{2}
$$

and the local relative trace formula as the equality between the expansions of

$$
\lim _{t \rightarrow \infty} \int_{\widetilde{H}(F)} \int_{N(F)} K_{f}(h, n) \psi(n) u(h, t) u(n, t) d n d h .
$$

In this local setting

$$
K_{f}(x, y)=\int_{\widetilde{G}(F)} f_{1}(g) f_{2}\left(x^{-1} g y\right) d g, \quad K_{f^{\prime}}(x, y)=\int_{\widetilde{G}^{\prime}(F)} f_{1}^{\prime}(g) f_{2}^{\prime}\left(x^{-1} g y\right) d g
$$

and $u(n, t)$ and $u(h, t)$ are truncation parameters that are needed due to convergence issues. They are defined analogously to Arthur's truncation [1991, Section 3].

We use the following ideas in this paper to rewrite these local trace formulas in terms of orbital integrals and representations:

- methods of Arthur [1991] from the local trace formula,

- methods of Flicker [1991], Jacquet [2005] and Ye [1989] from the relative trace formula,

- Harish-Chandra's Plancherel formula [Harish-Chandra 1984; Waldspurger 2003],

- Jacquet, Lapid and Rogawski's methods for regularizing period integrals [Jacquet et al. 1999; Jacquet $\geq 2012$ ]. 
The power of the two trace formulas lies in the comparison. For "matching functions", the geometric expansions of the two local relative trace formulas are equal. By comparing the spectral expansions in these two trace formulas, we get an analogue of (1-1), giving the following identity between local Bessel distributions for functions on $U(2)$ and local relative Bessel distributions for functions on $\operatorname{GL}(2, E)$, and therefore local periods and local Whittaker functionals:

Theorem 1.1. If $\sigma$ is a supercuspidal representation on $\widetilde{G}(F)$ that is the unstable base change lift of the supercuspidal representation $\sigma^{\prime}$ of $\widetilde{G}^{\prime}(F)$, and

$$
f^{\prime}=f_{1}^{\prime} \otimes f_{2}^{\prime} \in C_{c}^{\infty}\left(\widetilde{G}^{\prime}(F) \times \widetilde{G}^{\prime}(F)\right) \quad \text { and } \quad f=f_{1} \otimes f_{2} \in C_{c}^{\infty}(\widetilde{G}(F) \times \widetilde{G}(F))
$$

are matching functions, then

$$
\begin{aligned}
& d\left(\sigma^{\prime}\right) \sum_{S^{\prime} \in \mathscr{B}\left(\sigma^{\prime}\right)} W_{\sigma^{\prime}}^{\prime}\left(\sigma^{\prime}\left(f_{2}^{\prime}\right) S^{\prime} \sigma^{\prime}\left(f_{1}^{\prime \vee}\right)\right) \overline{W_{\sigma^{\prime}}^{\prime}\left(S^{\prime}\right)} \\
& =d(\sigma) \sum_{S \in \mathscr{P}(\sigma)} P_{\sigma}\left(\sigma\left(f_{2}\right) S \sigma\left(f_{1}^{\vee}\right)\right) \overline{W_{\sigma}(S)},
\end{aligned}
$$

where $d(\sigma)$ is the formal degree of $\sigma, \mathscr{B}(\sigma)$ is an orthonormal basis of the Hilbert space of Hilbert-Schmidt operators on $V_{\sigma}$,

$$
\begin{aligned}
W_{\sigma^{\prime}}^{\prime}\left(S^{\prime}\right) & =\int_{N^{\prime}(F)} \operatorname{tr}\left(\sigma^{\prime}(n) S^{\prime}\right) \psi^{\prime}\left(n^{-1}\right) d n, \\
W_{\sigma}(S) & =\int_{N(F)} \operatorname{tr}(\sigma(n) S) \psi\left(n^{-1}\right) d n, \\
P_{\sigma}(S) & =\int_{\widetilde{H}(F)} \operatorname{tr}(\sigma(h) S) d h .
\end{aligned}
$$

The Bessel and relative Bessel distributions $B_{\pi^{\prime}}^{\prime}\left(f^{\prime}\right)$ and $B_{\pi}(f)$ factor into local (relative) Bessel distributions $B_{\pi_{v}^{\prime}}^{\prime}\left(f_{v}^{\prime}\right)$ and $B_{\pi_{v}}\left(f_{v}\right)$, but it is not clear how to normalize the local distributions. The distributions on the left and right-hand side of (1-2) are each the product of two local distributions and (1-2) can be restated as

$$
d\left(\sigma^{\prime}\right) B_{\sigma^{\prime}}^{\prime}\left(f_{2}^{\prime}\right) B_{\sigma^{*}}^{\prime}\left(f_{1}^{\prime}\right)=d(\sigma) B_{\sigma}\left(f_{2}\right) B_{\sigma^{*}}\left(f_{1}\right) .
$$

We note that the local period integral $P_{\sigma}(S)$ is not a convergent integral if $\sigma$ is not a discrete series representation. To develop the local relative trace formula we have to define a local regularized period integral. Let $K=G\left(\mathcal{O}_{F}\right)$ and let $P=N M$. For $\lambda \in \mathbb{C}$ and $m=\left(\begin{array}{cc}\alpha & 0 \\ 0 & \beta\end{array}\right)$ let $e^{\lambda H_{M}(m)}=|\alpha / \beta|_{E}$ where $|\cdot|_{E}$ denotes the normalized valuation on $E$. For a principal series representation $\pi$ of $\widetilde{G}$ and $u, v \in \pi$ we define the matrix coefficient $f_{u, v}(g)=\langle\pi(g) u, v\rangle$. Asymptotically on $M, f_{u, v}$ will equal a finite sum of functions of the form $e^{\lambda H_{M}(m)}$. We define the regularized period 
integral as:

$$
\begin{aligned}
\int_{\widetilde{H}(F)}^{*} f_{u, v}(h) d h:= & \int_{\widetilde{H}(F)} f_{u, v}(h) u(h, t) d h \\
& +\int_{\widetilde{K}_{H} \times \widetilde{K}_{H}} \int_{\widetilde{M}_{H}^{+}(F)}^{\sharp} D_{P_{H}}(m) f_{u, v}\left(k_{1} m k_{2}\right)(1-u(m, t)) d m d k_{1} d k_{2}
\end{aligned}
$$

where

$$
\int_{\widetilde{M}_{H}^{+}(F)}^{\sharp} e^{\lambda H_{M}(m)}(1-u(m, t)) d m
$$

is the meromorphic continuation at $v=0$ of

$$
\int_{\widetilde{M}_{H}^{+}(F)} e^{(v+\lambda) H_{M}(m)}(1-u(m, t)) d m
$$

which is absolutely convergent for $\operatorname{Re}(v) \ll 0$.

We prove that the regularized period integral is an $H(F) \times H(F)$-invariant linear functional, and we relate it to the truncated period integral that initially appears in the local relative trace formula as follows. By abuse of notation we identify a character $\chi$ of $\tilde{M}(F)$ with a character $\chi$ of $E^{\times}$by letting $\chi\left(\begin{array}{ll}a & 0 \\ 0 & b\end{array}\right)=\chi(a) \chi^{-1}(b)$. For $\lambda \in \mathbb{C}$ we let $\chi_{\lambda}(m)=\chi(m) e^{\lambda\left(H_{M}(m)\right)}$. We let $I_{P}\left(\chi_{\lambda}\right)$ be the parabolically induced normalized representation acting on the Hilbert space $\mathscr{H}_{P}(\chi)$. Then for $S \in \mathscr{B}_{P}(\chi)$,

$$
\operatorname{tr}\left(I_{P}\left(\chi_{\lambda}, k_{1} g k_{2}\right) S\right)=E_{P}\left(g, \Psi_{S}, \lambda\right)_{k_{1}, k_{2}},
$$

where $E_{P}(g, \Psi, \lambda)$ is the Eisenstein integral and

$$
\left(C^{P} E_{P}\right)(m, \psi, \lambda)=\left(c_{P \mid P}(1, \lambda) \psi\right)(m) e^{\lambda H_{M}(m)}+\left(c_{P \mid P}(w, \lambda) \psi\right)(m) e^{-\lambda H_{M}(m)} .
$$

We fix a uniformizer $\varpi$ in $F$ (and $E$ ) and $q^{-1}=|\varpi|_{F}$.

Proposition 1.2. Fix a character $\chi$ of $E^{\times}$such that $\chi(\varpi)=1$. Then for $t \gg 0$,

$$
\begin{aligned}
& \int_{\widetilde{H}(F)} \operatorname{tr}\left(I_{P}\left(\chi_{\lambda}, h\right) S\right) u(h, t) d h= \int_{\widetilde{H}(F)}^{*} \operatorname{tr}\left(I_{P}\left(\chi_{\lambda}, h\right) S\right) d h \\
&-\delta(\chi)\left(1+q^{-1}\right)\left(\frac{q^{2 \lambda(t+1)}}{1-q^{2 \lambda}} \int_{\widetilde{K}_{H} \times \widetilde{K}_{H}} c(1, \lambda) \Psi_{S}(1)_{k_{1}, k_{2}} d k_{1} d k_{2}\right. \\
&\left.\quad+\frac{q^{-2 \lambda(t+1)}}{1-q^{-2 \lambda}} \int_{\widetilde{K}_{H} \times \widetilde{K}_{H}} c(w, \lambda) \Psi_{S}(1)_{k_{1}, k_{2}} d k_{1} d k_{2}\right),
\end{aligned}
$$

where $\delta(\chi)=1$ if $\left.\chi\right|_{O_{F}^{\times}}=1$ and $\delta(\chi)=0$ if $\left.\chi\right|_{\mathscr{O}_{F}^{\times}} \neq 1$.

Denote the action of the nontrivial element in $\operatorname{Gal}(E / F)$ on $x \in E$ by $\bar{x}$. Denote by $N_{E / F}$ the norm map from $E^{\times}$to $F^{\times}$. Let $E^{1}=\left\{x \in E^{\times}: N_{E / F}(x)=1\right\}$. Let $\eta$ denote an element in $G(F)$ such that $\bar{\eta}^{-1} \eta=\left(\begin{array}{ll}0 & 1 \\ 1 & 0\end{array}\right)$. 
We define

$$
D_{\chi_{\lambda}^{\prime}}^{\prime}\left(f^{\prime}\right)=\sum_{S^{\prime} \in \mathscr{B}_{P}\left(\chi^{\prime}\right)} W_{\chi_{\lambda}^{\prime}}^{\prime}\left(S_{\lambda}^{\prime}\left[f^{\prime}\right]\right) \overline{W_{\chi_{\lambda}^{\prime}}^{\prime}\left(S^{\prime}\right)} \quad \text { and } \quad D_{\chi_{\lambda}}(f)=\sum_{S \in \mathscr{B}_{P}(\chi)} P_{\chi_{\lambda}}\left(S_{\lambda}[f]\right) \overline{W_{\chi_{\lambda}}(S)} \text {, }
$$

where

$$
\begin{aligned}
W_{\chi_{\lambda}^{\prime}}^{\prime}\left(S^{\prime}\right) & =\lim _{t \rightarrow \infty} \int_{N^{\prime}(F)} \operatorname{tr}\left(I_{P^{\prime}}\left(\chi_{\lambda}^{\prime}, n\right) S^{\prime}\right) \psi^{\prime}\left(n^{-1}\right) u(n, t) d n, \\
W_{\chi_{\lambda}}(S) & =\lim _{t \rightarrow \infty} \int_{N(F)} \operatorname{tr}\left(I_{P}\left(\chi_{\lambda}, n\right) S\right) \psi\left(n^{-1}\right) u(n, t) d n, \\
P_{\chi_{\lambda}}(S) & =\int_{\widetilde{H}(F)}^{*} \operatorname{tr}\left(I_{P}\left(\chi_{\lambda}, h\right) S\right) d h, \\
S_{\lambda}[f] & =I_{P}\left(\chi_{\lambda}, f_{2}\right) S I_{P}\left(\chi_{\lambda}, f_{1}^{\vee}\right) .
\end{aligned}
$$

We let $\Pi_{2}\left(\widetilde{G}^{\prime}(F)\right)$ be a set of equivalence classes of irreducible, tempered square integrable representations of $\widetilde{G}^{\prime}(F)$. We identify unitary characters on $\widetilde{M}^{\prime}(F)$ with characters on $E^{\times}$that are trivial on $E^{1}$. We let $\left\{\Pi_{2}\left(\tilde{M}^{\prime}(F)\right)\right\}$ be a set of representatives of unitary characters $\chi^{\prime}$ on $\tilde{M}^{\prime}(F)$ such that $\chi^{\prime}(\varpi)=1$. We let $\mu\left(\chi_{\lambda}^{\prime}\right)$ be Harish-Chandra's $\mu$-function. We take the analogous definitions for $\widetilde{G}(F)$.

Theorem 1.3 (local Kuznetsov trace formula). For any

$$
f^{\prime}=f_{1}^{\prime} \otimes f_{2}^{\prime} \in C_{c}^{\infty}\left(\widetilde{G}^{\prime}(F) \times \widetilde{G}^{\prime}(F)\right),
$$

we have

$$
\begin{aligned}
\lim _{t \rightarrow \infty} & \int_{N^{\prime}(F)} \int_{N^{\prime}(F)} K_{f^{\prime}}\left(n_{1}, n_{2}\right) \psi^{\prime}\left(n_{1}^{-1} n_{2}\right) u\left(n_{1}, t\right) u\left(n_{2}, t\right) d n_{1} d n_{2} \\
& =\int_{a \in E^{\times} / E^{1}} O^{\prime}\left(f_{1}, \psi^{\prime}, a\right) O^{\prime}\left(f_{2}^{\prime}, \bar{\psi}^{\prime}, a\right)|a|_{E} d^{\times} a \\
& =\sum_{\sigma^{\prime} \in \Pi_{2}\left(\widetilde{G}^{\prime}(F)\right)} d\left(\sigma^{\prime}\right) D_{\sigma^{\prime}}^{\prime}\left(f^{\prime}\right)+\frac{1}{2} \sum_{\chi^{\prime} \in\left\{\Pi_{2}\left(\widetilde{M}^{\prime}(F)\right)\right\}} d\left(\chi^{\prime}\right) \int_{0}^{\pi i / \log q} \mu\left(\chi_{\lambda}^{\prime}\right) D_{\chi_{\lambda}^{\prime}}^{\prime}\left(f^{\prime}\right) d \lambda,
\end{aligned}
$$

where

$$
O^{\prime}\left(f_{i}^{\prime}, \psi^{\prime}, a\right)=\int_{N^{\prime}(F)} \int_{N^{\prime}(F)} f_{i}^{\prime}\left(n_{1}^{-1}\left(\begin{array}{cc}
0 & 1 \\
-1 & 0
\end{array}\right)\left(\begin{array}{cc}
a & 0 \\
0 & \bar{a}^{-1}
\end{array}\right) n_{2}\right) \overline{\psi^{\prime}\left(n_{1}^{-1} n_{2}\right)} d n_{1} d n_{2} .
$$

Theorem 1.4 (local relative trace formula). For any

$$
f=f_{1} \otimes f_{2} \in C_{c}^{\infty}(\widetilde{G}(F) \times \widetilde{G}(F)),
$$

we have 


$$
\begin{aligned}
& \lim _{t \rightarrow \infty} \int_{\widetilde{H}(F)} \int_{N(F)} K_{f}(h, n) \psi(n) u(h, t) u(n, t) d n d h \\
&= \int_{a \in E^{\times} / E^{1}} O\left(f_{1}, \psi, a\right) O\left(f_{2}, \bar{\psi}, a\right)|a|_{E} d^{\times} a \\
&= \sum_{\sigma \in \Pi_{2}(\widetilde{G}(F))} d(\sigma) D_{\sigma}(f)+\frac{1}{2} \sum_{\substack{\chi \in\left\{\Pi_{2}(\widetilde{M}(F))\right\} \\
\chi^{2} \neq 1,\left.\chi\right|_{F} \times 1}} \widetilde{D}_{\chi}(f) \\
&+\frac{1}{2} \sum_{\substack{\left.\chi \in\left\{\Pi_{2}(\widetilde{M}(F))\right\} \\
\chi\right|_{E^{1}}=1}} d(\chi) \int_{0}^{\pi i / \log q} \mu\left(\chi_{\lambda}\right) D_{\chi_{\lambda}}(f) d \lambda
\end{aligned}
$$

where

$$
O\left(f_{i}, \psi, a\right)=\int_{\widetilde{H}(F)} \int_{N(F)} f_{i}\left(h^{-1} \eta\left(\begin{array}{ll}
a & 0 \\
0 & 1
\end{array}\right) n\right) \overline{\psi(n)} d n d h .
$$

The representations that occur on the right-hand side of Theorem 1.4 are exactly the representations that are in the image of the unstable base change lift on $\widetilde{G}^{\prime}(F)$. The additional discrete term $\widetilde{D}_{\chi}(f)$ corresponds to the representations that lift from discrete series on $\widetilde{G}^{\prime}(F)$ to principal series on $\widetilde{G}(F)$.

In addition to the spectral comparison, these local trace formulas also have applications on the geometric side. If we define the inner product of two functions $g_{1}, g_{2}$ on $E^{\times} / E^{1}$ by

$$
\left\langle g_{1}, g_{2}\right\rangle=\int_{a \in E^{\times} / E^{1}} g_{1}(a) g_{2}(a)|a|_{E} d^{\times} a,
$$

then:

Proposition 1.5 (orthogonality relations). For $f_{1}$ and $f_{2}$ matrix coefficients of the supercuspidal representations $\sigma_{1}$ and $\sigma_{2}$ of $\widetilde{G}(F)$ and $f_{1}^{\prime}$ and $f_{2}^{\prime}$ matrix coefficients of the supercuspidal representations $\sigma_{1}^{\prime}$ and $\sigma_{2}^{\prime}$ of $\widetilde{G}^{\prime}(F)$,

$$
\begin{aligned}
\left\langle O^{\prime}\left(f_{1}^{\prime}, \psi^{\prime}, \cdot\right), O^{\prime}\left(f_{2}^{\prime}, \psi^{\prime-1}, \cdot\right)\right\rangle & \neq 0 \Longleftrightarrow \sigma_{1}^{\prime} \sim \sigma_{2}^{\prime}, \\
\left\langle O\left(f_{1}, \psi, \cdot\right), O\left(f_{2}, \psi^{-1}, \cdot\right)\right\rangle & \neq 0 \Longleftrightarrow \sigma_{1} \sim \sigma_{2} .
\end{aligned}
$$

The rest of this paper is organized as follows. In Section 2 we define notation and give normalizations of measures. In Section 3 we develop the local Kuznetsov trace formula. For the geometric expansion we rewrite our trace formula in terms of orbital integrals corresponding to the $N^{\prime} \backslash G^{\prime} / N^{\prime}$ double cosets. The orbital integrals for $f_{1}^{\prime}$ and $f_{2}^{\prime}$ initially depend on the truncation and are intertwined. It is only through the multiplication of the two orbital integrals, integration over the space of double cosets, and the nontriviality of the character $\psi^{\prime}$, that we are able to untangle the orbital integral for $f_{1}^{\prime}$ from the orbital integral for $f_{2}^{\prime}$. For the spectral 
expansion we apply Harish-Chandra's Plancherel formula to rewrite the local kernel in terms of representations. We are left with truncated integrals over the unipotent subgroup of matrix coefficients against the character $\psi^{\prime}$. By the smoothness of the matrix coefficients and the appearance of the character, we show these distributions stabilize for $t$ large.

In Section 4 we develop the local relative trace formula of $H \backslash G / N$. In the spectral expansion we have truncated integrals of matrix coefficients over $H$ that do not converge without the truncation. We define the regularized period integral $P_{\chi_{\lambda}}(S)$. We use the asymptotics of matrix coefficients of tempered representations to prove the truncated integral is a polynomial exponential function in the truncation parameter $t$. We define the regularized integral as the constant term of this polynomial, and prove that this is an $H \times H$ invariant linear functional and the relevant term in the local relative trace formula.

In Section 5 we compare our two local trace formulas. There is a bijection between the "admissible" $N^{\prime} \backslash G^{\prime} / N^{\prime}$ cosets and the "admissible" $H \backslash G / N$ cosets and both of these sets can be parametrized by $E^{\times} / E^{1}$. This bijection allows us to compare the geometric sides. By work of Ye and Flicker, we know that for any $f^{\prime}$ there is an $f$ such that the orbital integrals are equal for corresponding cosets. Thus, by their geometric expansions, our local trace formulas are equal for matching functions. This gives an equality of the spectral expansions and of local distributions.

This paper would not have come into being had it not been for my teacher and advisor, Jonathan Rogawski. These thoughts originated as my PhD thesis under his direction, and his ideas, support, and guidance were critical to its completion. I am fortunate and will be forever grateful to have had him as a mentor. He could explain complicated math in a clear and simple way that aimed at the heart of the problem. He served, and continues to serve, as the role model of the inquisitive, patient, and approachable mathematician.

\section{Notation}

Let $F$ be a non-Archimedean local field of characteristic 0 and odd residual characteristic $q$. Let $E$ be an unramified quadratic extension of $F$. Let $O_{F}$ and $O_{E}$ denote the rings of integers in $F$ and $E$, respectively. Let $\varpi$ denote a uniformizer in the maximal ideal of $O_{F}$. Thus $\varpi$ is also a uniformizer in $E$. Let $v(\cdot)$ denote the valuation on $F$, extended to $E$. Let $|\cdot|_{F}$ and $|\cdot|_{E}$ denote the normalized valuations on $F E$, respectively. Thus for $a \in F^{\times},|a|_{E}=|a|_{F}^{2}$. Denote the action of the nontrivial element in $\operatorname{Gal}(E / F)$ on $x \in E$ by $\bar{x}$. Denote by $N_{E / F}$ the norm map from $E^{\times}$to $F^{\times}$. Let $E^{1}=\left\{a \in E^{\times}: N_{E / F}(a)=1\right\}$. 
Let $H=\mathrm{GL}(2) / F$ and let $G=\operatorname{Res}_{E / F} H$, the restriction of scalars of GL(2) from $E$ to $F$. Thus $G(F)=\mathrm{GL}(2, E)$. Let

$$
G^{\prime}=\mathrm{U}(2, F)=\left\{g \in G:^{t} \bar{g}\left(\begin{array}{rr}
0 & 1 \\
-1 & 0
\end{array}\right) g=\left(\begin{array}{rr}
0 & 1 \\
-1 & 0
\end{array}\right)\right\} .
$$

We note that by defining the quasisplit unitary group in this way, $S L(2, F) \subset G^{\prime}(F)$. Let $N^{\prime}$ and $N$ be the upper triangular unipotent matrices of $G^{\prime}$ and $G$, respectively. Let $M^{\prime}$ and $M$ be the diagonal subgroups of $G^{\prime}$ and $G$, respectively. That is,

$$
M^{\prime}(F)=\left\{\left(\begin{array}{cc}
a & 0 \\
0 & \bar{a}^{-1}
\end{array}\right): a \in E^{\times}\right\} \quad \text { and } \quad M(F)=\left\{\left(\begin{array}{cc}
a & 0 \\
0 & b
\end{array}\right): a, b \in E^{\times}\right\} .
$$

Occasionally by abuse of notation we let $n=\left(\begin{array}{ll}1 & n \\ 0 & 1\end{array}\right)$ and $a=\left(\begin{array}{cc}a & 0 \\ 0 & \bar{a}^{-1}\end{array}\right)$. Let $P=N M$ and $P^{\prime}=N^{\prime} M^{\prime}$. Let $K=G\left(\mathscr{O}_{F}\right)$ and $K^{\prime}=G^{\prime}\left(\mathscr{O}_{F}\right)$. Let $Z$ and $Z^{\prime}$ denote the centers of $G$ and $G^{\prime}$, respectively. For any subgroup $X$ of $G$ let $\tilde{X}=Z \cap X \backslash X$ and $X_{H}=X \cap H$. By abuse of notation we identify a character $\chi$ of $\tilde{M}(F)$ with a character $\chi$ of $E^{\times}$by letting $\chi\left(\begin{array}{ll}a & 0 \\ 0 & b\end{array}\right)=\chi(a) \chi^{-1}(b)$.

Let $\psi^{\prime}$ be an additive character on $F$ with conductor $O_{F}$. Let $\psi$ be the additive character on $E$ defined by $\psi(x)=\psi^{\prime}(x+\bar{x})$. By abuse of notation we will also denote by $\psi$ and $\psi^{\prime}$ the corresponding characters on $N(F)$ and $N^{\prime}(F)$, respectively. Let $f=f_{1} \otimes f_{2} \in C_{c}^{\infty}(\widetilde{G}(F) \times \widetilde{G}(F))$ and $f^{\prime}=f_{1}^{\prime} \otimes f_{2}^{\prime} \in C_{c}^{\infty}\left(\widetilde{G}^{\prime}(F) \times \widetilde{G}^{\prime}(F)\right)$. For a function $f$ on $G$, let $f^{\vee}(g)=f\left(g^{-1}\right)$.

To define the local Kuznetsov trace formula and local relative trace formula we first multiply our function by the characteristic function of a large compact subset of $\widetilde{G}(F)$ via Arthur's local truncation [1991, §3], and then take the limit of the integral of the truncated function. For $g \in G(F), t \in \mathbb{Z}^{+}$, let

$$
u(g, t)= \begin{cases}1 & \text { if } g=z k_{1}\left(\begin{array}{ll}
1 & 0 \\
0 & \alpha
\end{array}\right) k_{2}, \text { for some } k_{1}, k_{2} \in K, z \in Z(F), 0 \leq v(\alpha) \leq t, \\
0 & \text { otherwise. }\end{cases}
$$

We note that $u(\cdot, t)$ is well-defined on $\widetilde{G}(F)$ and

$$
u\left(\left(\begin{array}{ll}
1 & x \\
0 & 1
\end{array}\right), t\right)= \begin{cases}1 & \text { if } x \in \varpi^{[-t / 2]} \mathscr{O}_{E}, \\
0 & \text { otherwise }\end{cases}
$$

where $[x]$ is the integral part of $x$.

If $X$ is a closed subgroup of $\widetilde{G}(F)$ with the subgroup topology, $\operatorname{supp}(u(\cdot, t)) \cap X$ is a compact set.

We normalize the Haar measure $d x$ on $F$ so that $\operatorname{vol}\left(O_{F}\right)=1$. We define the multiplicative measure $d^{\times} x$ on $F^{\times}$as

$$
d^{\times} x=\frac{1}{1-q^{-1}} \frac{1}{|x|_{F}} d x .
$$


Thus $\operatorname{vol}\left(0_{F}^{\times}\right)=1$. We let $N(F)$ and $M(F)$ have the measures induced by $d x$ and $d^{\times} x$. We normalize the Haar measure $d k$ on $K$ so that $\operatorname{vol}(K)=1$. We define the measure $d g$ on $G(F)$ by

$$
\int_{G(F)} f(g) d g=\int_{M(F)} \int_{N(F)} \int_{K} f(m n k) d k d n d m .
$$

We define $d g^{\prime}$ on $G^{\prime}(F)$ similarly. We normalize Haar measure on $\widetilde{K}$ by taking $\operatorname{vol}(\widetilde{K})=1$.

We let $d^{\times} a$ be the unique Haar measure on $E^{\times} / E^{1}$ such that

$$
\operatorname{vol}\left(\mathscr{O}_{E}^{\times} / E^{1}\right)=\frac{1}{1+q^{-1}}
$$

\section{The local Kuznetsov trace formula for $\mathrm{U}(2)$}

In this section we develop a local Kuznetsov trace formula for the quasisplit unitary group in two variables. We expand this local Kuznetsov trace formula geometrically in terms of separate orbital integrals for $f_{1}^{\prime}$ and $f_{2}^{\prime}$. Then we use Harish-Chandra's Plancherel formula to rewrite this expression spectrally in terms of representations.

We define the local Kuznetsov trace formula for

$$
f^{\prime}=f_{1}^{\prime} \otimes f_{2}^{\prime} \in C_{c}^{\infty}\left(\widetilde{G}^{\prime}(F) \times \widetilde{G}^{\prime}(F)\right)
$$

as the equality between the geometric and spectral expansions of

$$
\lim _{t \rightarrow \infty} \int_{\left(N^{\prime} \times N^{\prime}\right)(F)} K_{f^{\prime}}\left(n_{1}, n_{2}\right) \psi\left(n_{1}^{-1} n_{2}\right) u\left(n_{1}, t\right) u\left(n_{2}, t\right) d n_{1} d n_{2}
$$

where

$$
K_{f^{\prime}}\left(n_{1}, n_{2}\right)=\int_{\widetilde{G}^{\prime}(F)} f_{1}^{\prime}(g) f_{2}^{\prime}\left(n_{1}^{-1} g n_{2}\right) d g .
$$

We will show that for a fixed $f^{\prime}$ this limit stabilizes, that is, there exists a $T$ such that for all $t^{\prime} \geq T$,

$$
\begin{aligned}
\int_{\left(N^{\prime} \times N^{\prime}\right)(F)} K_{f^{\prime}}\left(n_{1}, n_{2}\right) \psi^{\prime}\left(n_{1}^{-1} n_{2}\right) u\left(n_{1}, t^{\prime}\right) u\left(n_{2}, t^{\prime}\right) d n_{1} d n_{2} \\
\quad=\lim _{t \rightarrow \infty} \int_{\left(N^{\prime} \times N^{\prime}\right)(F)} K_{f^{\prime}}\left(n_{1}, n_{2}\right) \psi\left(n_{1}^{-1} n_{2}\right) u\left(n_{1}, t\right) u\left(n_{2}, t\right) d n_{1} d n_{2} .
\end{aligned}
$$

3A. The geometric expansion. In this subsection we rewrite

$$
\lim _{t \rightarrow \infty} \int_{N^{\prime}(F)} \int_{N^{\prime}(F)} K_{f^{\prime}}\left(n_{1}, n_{2}\right) \psi^{\prime}\left(n_{1}^{-1} n_{2}\right) u\left(n_{1}, t\right) u\left(n_{2}, t\right) d n_{1} d n_{2}
$$

as an integral over admissible cosets of a product of an orbital integral for $f_{1}^{\prime}$ and an orbital integral for $f_{2}^{\prime}$. 
3A1. Integration formula. Let $w=\left(\begin{array}{rr}0 & 1 \\ -1 & 0\end{array}\right)$. For $a \in E^{\times}$, let $\beta_{a}=\left(\begin{array}{cc}a & 0 \\ 0 & \bar{a}^{-1}\end{array}\right)$ and $\gamma_{a}=w\left(\begin{array}{cc}a & 0 \\ 0 & \bar{a}^{-1}\end{array}\right)$. By the Bruhat decomposition, $G^{\prime}=P^{\prime} \sqcup P^{\prime} w P^{\prime}$. Thus

$\left\{\beta_{a}: \begin{array}{l}a \text { is in a set of } \\ \text { representatives for } E^{\times} / E^{1}\end{array}\right\} \cup\left\{\gamma_{a}: \begin{array}{l}a \text { is in a set of } \\ \text { representatives for } E^{\times} / E^{1}\end{array}\right\}$ is a set of representatives for the double cosets of $N^{\prime}(F) \backslash \widetilde{G}^{\prime}(F) / N^{\prime}(F)$.

For $g \in G^{\prime}(F)$ let

$C_{g}\left(N^{\prime}(F) \times N^{\prime}(F)\right)$

$$
=\left\{\left(n_{1}, n_{2}\right) \in N^{\prime}(F) \times N^{\prime}(F): n_{1}^{-1} g n_{2}=z g \text { for some } z \in Z^{\prime}(F)\right\} .
$$

Definition 3.1. An element $g \in \widetilde{G}^{\prime}(F)$ and its corresponding orbit are called $a d$ missible if the map

$$
C_{g}\left(N^{\prime}(F) \times N^{\prime}(F)\right) \rightarrow \mathbb{C}:\left(n_{1}, n_{2}\right) \mapsto \psi^{\prime}\left(n_{1}^{-1} n_{2}\right)
$$

is trivial.

By a simple calculation we see that

$$
\begin{aligned}
& C_{\beta_{a}}\left(N^{\prime}(F) \times N^{\prime}(F)\right)=\left\{\left(\left(\begin{array}{ll}
1 & x \\
0 & 1
\end{array}\right),\left(\begin{array}{cc}
1 & \frac{x}{a \bar{a}} \\
0 & 1
\end{array}\right)\right): x \in F\right\}, \\
& C_{\gamma_{a}}\left(N^{\prime}(F) \times N^{\prime}(F)\right)=1 .
\end{aligned}
$$

Thus the orbits represented by $\left\{\beta_{1}\right\} \cup\left\{\gamma_{a}: a \in E^{\times} / E^{1}\right\}$ are admissible.

We use the following integration formula to rewrite $K_{f^{\prime}}\left(n_{1}, n_{2}\right)$ as an integral over the admissible cosets. Unlike in the global case the trivial admissible coset, $\beta_{1}$, will not contribute to the trace formula.

For any $F \in C_{c}\left(\widetilde{G}^{\prime}(F)\right)$,

$$
\int_{\widetilde{G}^{\prime}(F)} F(g) d g=\int_{E^{\times} / E^{1}} \int_{\left(N^{\prime} \times N^{\prime}\right)(F)} F\left(n_{1}^{-1} \gamma_{a} n_{2}\right) d n_{1} d n_{2}|a|_{E} d^{\times} a .
$$

3A2. Separating the orbital integrals. Let

$$
K^{t}\left(f^{\prime}\right)=\int_{N^{\prime}(F)} \int_{N^{\prime}(F)} K_{f^{\prime}}\left(n_{1}, n_{2}\right) \psi\left(n_{1}^{-1} n_{2}\right) u\left(n_{1}, t\right) u\left(n_{2}, t\right) d n_{1} d n_{2} .
$$

Clearly $K^{t}\left(f^{\prime}\right)$ is absolutely convergent because $f_{1}^{\prime}$ and $u(\cdot, t)$ have compact support on $\widetilde{G}^{\prime}(F)$ and $N^{\prime}(F)$ respectively. By changing the order of integration and using (3-1), we see that $K^{t}\left(f^{\prime}\right)$ equals

$$
\begin{aligned}
& \int_{E^{\times} / E^{1}} \int_{\left(N^{\prime} \times N^{\prime}\right)(F)} \int_{\left(N^{\prime} \times N^{\prime}\right)(F)} f_{1}^{\prime}\left(\hat{n}_{1}^{-1} \gamma_{a} \hat{n}_{2}\right) f_{2}^{\prime}\left(n_{1}^{-1} \hat{n}_{1}^{-1} \gamma_{a} \hat{n}_{2} n_{2}\right) \\
& \times \psi^{\prime}\left(n_{1}^{-1} n_{2}\right) u\left(n_{1}, t\right) u\left(n_{2}, t\right) d n_{1} d n_{2} d \hat{n}_{1} d \hat{n}_{2}|a|_{E} d^{\times} a .
\end{aligned}
$$


This integral is absolutely convergent because the map

$$
N^{\prime}(F) \times E^{\times} / E^{1} \times N^{\prime}(F) \rightarrow \widetilde{G}^{\prime}(F)
$$

defined by

$$
\left(n_{1}, a, n_{2}\right) \mapsto n_{1}^{-1} \gamma_{a} n_{2}
$$

is injective and $f_{1}^{\prime}$ has compact support. By a change of variables we have

$$
K^{t}\left(f^{\prime}\right)=\int_{E^{\times} / E^{1}} K^{t}\left(\gamma_{a}, f^{\prime}\right)|a|_{E} d^{\times} a
$$

where

$$
\begin{array}{r}
K^{t}\left(\gamma_{a}, f^{\prime}\right)=\int_{\left(N^{\prime} \times N^{\prime}\right)(F)} \int_{\left(N^{\prime} \times N^{\prime}\right)(F)} f_{1}^{\prime}\left(\hat{n}_{1}^{-1} \gamma_{a} \hat{n}_{2}\right) f_{2}^{\prime}\left(n_{1}^{-1} \gamma_{a} n_{2}\right) \psi^{\prime}\left(n_{1}^{-1} \hat{n}_{1} \hat{n}_{2}^{-1} n_{2}\right) \\
\times u\left(\hat{n}_{1}^{-1} n_{1}, t\right) u\left(\hat{n}_{2}^{-1} n_{2}, t\right) d n_{1} d n_{2} d \hat{n}_{1} d \hat{n}_{2} .
\end{array}
$$

To complete the geometric expansion of the local Kuznetsov trace formula we rewrite $K^{t}\left(f^{\prime}\right)$ for $t \gg 0$ as an integral of two separate orbital integrals. We begin by examining the dependence of the integrand on the truncation.

Lemma 3.2. Let $f_{1}^{\prime}, f_{2}^{\prime} \in C_{c}\left(\widetilde{G}^{\prime}(F)\right)$. For each $t_{0}>0$ there exists a $T>0$ such that for all $t \geq T$,

$$
\begin{aligned}
f_{1}^{\prime}\left(x_{1}^{-1} \gamma y_{1}\right) f_{2}^{\prime}\left(x_{2}^{-1} \gamma y_{2}\right) u\left(x_{1}^{-1} x_{2}, t\right) u\left(y_{1}^{-1} y_{2}, t_{0}\right) \\
\quad=f_{1}^{\prime}\left(x_{1}^{-1} \gamma y_{1}\right) f_{2}^{\prime}\left(x_{2}^{-1} \gamma y_{2}\right) u\left(y_{1}^{-1} y_{2}, t_{0}\right)
\end{aligned}
$$

for all $x_{1}, x_{2}, y_{1}, y_{2}, \gamma \in \widetilde{G}^{\prime}(F)$.

Proof. Let

$$
\Omega_{1}=\operatorname{supp}\left(f_{1}^{\prime}\right), \quad \Omega_{2}=\operatorname{supp}\left(f_{2}^{\prime}\right), \quad \Omega_{3}=\operatorname{supp}\left(u\left(\cdot, t_{0}\right)\right) \cap \widetilde{G}^{\prime}(F) .
$$

These sets are all compact on $\widetilde{G}^{\prime}(F)$. If $f_{1}^{\prime}\left(x_{1}^{-1} \gamma y_{1}\right) f_{2}^{\prime}\left(x_{2}^{-1} \gamma y_{2}\right) u\left(y_{1}^{-1} y_{2}, t_{0}\right) \neq 0$, then the following conditions must hold:

- $x_{1}^{-1} \in \Omega_{1} y_{1}^{-1} \gamma^{-1}$.

- $x_{2} \in \gamma y_{2} \Omega_{2}^{-1}$.

- $y_{1}^{-1} y_{2} \in \Omega_{3}$.

Thus if $f_{1}^{\prime}\left(x_{1}^{-1} \gamma y_{1}\right) f_{2}^{\prime}\left(x_{2}^{-1} \gamma y_{2}\right) u\left(y_{1}^{-1} y_{2}, t_{0}\right) \neq 0$, then $x_{1}^{-1} x_{2} \in \Omega_{1} \Omega_{3} \Omega_{2}^{-1}$. Because this is a compact set, there exists a $T>0$ such that $\Omega_{1} \Omega_{3} \Omega_{2}^{-1} \subseteq \operatorname{supp}(u(g, T))$. The lemma now follows. 
Now we use this lemma, along with the character $\psi^{\prime}$, to separate the two orbital integrals. By abuse of notation, in the proof of the following lemma we let

$$
\varpi^{n}=\left(\begin{array}{cc}
\varpi^{n} & 0 \\
0 & \varpi^{-n}
\end{array}\right) \quad \text { and } \quad a=\left(\begin{array}{cc}
a & 0 \\
0 & \bar{a}^{-1}
\end{array}\right)
$$

Lemma 3.3. For $f^{\prime}=f_{1}^{\prime} \otimes f_{2}^{\prime} \in C_{c}^{\infty}\left(\widetilde{G}^{\prime}(F) \times \widetilde{G}^{\prime}(F)\right)$, there exists a $T$ such that for all $t \geq T$ and $n \in \mathbb{Z}$,

$$
\int_{a \in \varpi^{n} \mathscr{O}_{E}^{\times} / E^{1}} K^{t}\left(\gamma_{a}, f^{\prime}\right) d^{\times} a=\int_{a \in \varpi^{n} \mathscr{O}_{E}^{\times} / E^{1}} O^{\prime}\left(f_{1}^{\prime}, \psi^{\prime}, a\right) O^{\prime}\left(f_{2}^{\prime}, \bar{\psi}^{\prime}, a\right) d^{\times} a
$$

where

$$
O^{\prime}\left(f^{\prime}, \psi^{\prime}, a\right)=\int_{N^{\prime}(F)} \int_{N^{\prime}(F)} f^{\prime}\left(n_{1}^{-1} \gamma_{a} n_{2}\right) \overline{\psi^{\prime}\left(n_{1}^{-1} n_{2}\right)} d n_{1} d n_{2} .
$$

Proof. We show that there is a hidden truncation on the right-hand side of (3-2) that comes from the fact that the two orbital integrals are simultaneously evaluated at the same $\gamma_{a}$. Let $K_{1}$ be an open compact subgroup of $\widetilde{G}^{\prime}(F)$ such that $f_{1}^{\prime}$ and $f_{2}^{\prime}$ are bi- $K_{1}$-invariant. There exists a positive constant $c$ such that

$$
\left(\begin{array}{cc}
a & 0 \\
0 & \bar{a}^{-1}
\end{array}\right) \in K_{1} \quad \text { for all } a \in\left(1+\varpi^{c} \mathscr{O}_{E}\right) E^{1}
$$

By definition

$$
\begin{aligned}
& \int_{a \in \varpi^{n} \Theta_{E}^{\times} / E^{1}} K^{t}\left(\gamma_{a}, f^{\prime}\right) d^{\times} a \\
& =\int_{a \in O_{E}^{\times} / E^{1}} \int_{\left(N^{\prime} \times N^{\prime}\right)(F)} f_{1}^{\prime}\left(\hat{n}_{1}^{-1} w \varpi^{n} a \hat{n}_{2}\right) \psi^{\prime}\left(\hat{n}_{1} \hat{n}_{2}^{-1}\right) \int_{\left(N^{\prime} \times N^{\prime}\right)(F)} f_{2}^{\prime}\left(n_{1}^{-1} w \varpi^{n} a n_{2}\right) \\
& \times \psi^{\prime}\left(n_{1}^{-1} n_{2}\right) u\left(\hat{n}_{1}^{-1} n_{1}, t\right) u\left(\hat{n}_{2}^{-1} n_{2}, t\right) d n_{2} d n_{1} d \hat{n}_{2} d \hat{n}_{1} d^{\times} a \\
& =\sum_{\eta \in \mathbb{O}_{E}^{\times} /\left(1+\varpi^{c} \mathscr{O}_{E}\right) E^{1}} \int_{a \in\left(1+\varpi^{c} \mathscr{O}_{E}\right) E^{1} / E^{1}} \int_{\left(N^{\prime} \times N^{\prime}\right)(F)} f_{1}^{\prime}\left(\hat{n}_{1}^{-1} w \varpi^{n} \eta a \hat{n}_{2}\right) \psi^{\prime}\left(\hat{n}_{1} \hat{n}_{2}^{-1}\right) \\
& \quad \times \int_{\left(N^{\prime} \times N^{\prime}\right)(F)} f_{2}^{\prime}\left(n_{1}^{-1} w \varpi^{n} \eta a n_{2}\right) \psi^{\prime}\left(n_{1}^{-1} n_{2}\right) u\left(\hat{n}_{1}^{-1} n_{1}, t\right) \\
& \times u\left(\hat{n}_{2}^{-1} n_{2}, t\right) d n_{2} d n_{1} d \hat{n}_{2} d \hat{n}_{1} d^{\times} a .
\end{aligned}
$$


By a change of variables and the fact that $f^{\prime}$ is locally constant the right-hand side of this equation is equal to

$\sum_{\eta \in \mathbb{O}_{E}^{\times} /\left(1+\varpi^{c} \mathscr{C}_{E}\right) E^{1}} \int_{\left(N^{\prime} \times N^{\prime}\right)(F)} f_{1}^{\prime}\left(\hat{n}_{1}^{-1} w \varpi^{n} \eta \hat{n}_{2}\right) \psi^{\prime}\left(\hat{n}_{1}\right)$

$$
\begin{aligned}
& \times \int_{\left(N^{\prime} \times N^{\prime}\right)(F)} f_{2}^{\prime}\left(n_{1}^{-1} w \varpi^{n} \eta n_{2}\right) \psi^{\prime}\left(n_{1}^{-1}\right) u\left(\hat{n}_{1}^{-1} n_{1}, t\right) d n_{1} d \hat{n}_{1} \\
& \quad \times \int_{a \in\left(1+\omega^{c} \mathscr{O}_{E}\right) E^{1} / E^{1}} \psi^{\prime}\left(a^{-1} \hat{n}_{2}^{-1} n_{2} a\right) u\left(a^{-1} \hat{n}_{2}^{-1} n_{2} a, t\right) d^{\times} a d n_{2} d \hat{n}_{2} .
\end{aligned}
$$

We can rewrite the inner integral as

$$
\begin{aligned}
u\left(\hat{n}_{2}^{-1} n_{2}, t\right) \int_{a \in\left(1+\varpi^{c} \mathscr{C}_{E}\right) E^{1} / E^{1}} \psi^{\prime}\left(\left(n_{2}-\hat{n}_{2}\right)(a \bar{a})^{-1}\right) d^{\times} a \\
=u\left(\hat{n}_{2}^{-1} n_{2}, t\right) \int_{b \in 1+\varpi^{c} \mathscr{C}_{F}} \psi^{\prime}\left(b\left(n_{2}-\hat{n}_{2}\right)\right) d^{\times} b \\
=u\left(\hat{n}_{2}^{-1} n_{2}, t\right) \frac{1}{1-q^{-1}} \psi^{\prime}\left(n_{2}-\hat{n}_{2}\right) \int_{b \in \varpi^{c} \widehat{C}_{F}} \psi^{\prime}\left(b\left(n_{2}-\hat{n}_{2}\right)\right) d b \\
=u\left(\hat{n}_{2}^{-1} n_{2}, t\right) u\left(\hat{n}_{2}^{-1} n_{2}, 2 c\right) \frac{\operatorname{vol}\left(\varpi^{c} \mathscr{O}_{F}\right)}{1-q^{-1}} \psi^{\prime}\left(\hat{n}_{2}^{-1} n_{2}\right) .
\end{aligned}
$$

Thus for $t \geq 2 c$,

$$
\begin{aligned}
& \int_{a \in \varpi^{n} \Theta_{E}^{\times} / E^{1}} K^{t}\left(\gamma_{a}, f^{\prime}\right) d^{\times} a=\int_{a \in \varpi^{n} \mathbb{O}_{E}^{\times} / E^{1}} \int_{\left(N^{\prime} \times N^{\prime}\right)(F)} f_{1}^{\prime}\left(\hat{n}_{1}^{-1} \gamma_{a} \hat{n}_{2}\right) \psi^{\prime}\left(\hat{n}_{1} \hat{n}_{2}^{-1}\right) \\
& \times \int_{\left(N^{\prime} \times N^{\prime}\right)(F)} f_{2}^{\prime}\left(n_{1}^{-1} \gamma_{a} n_{2}\right) \psi^{\prime}\left(n_{1}^{-1} n_{2}\right) u\left(\hat{n}_{1}^{-1} n_{1}, t\right) u\left(\hat{n}_{2}^{-1} n_{2}, 2 c\right) d n_{2} d n_{1} d \hat{n}_{2} d \hat{n}_{1} d^{\times} a .
\end{aligned}
$$

By Lemma 3.2 there exists a $T>0$ such that for all $t \geq \max \{T, 2 c\}$,

$$
\begin{aligned}
& \int_{a \in \varpi^{n} \Theta_{E}^{\times} / E^{1}} K^{t}\left(\gamma_{a}, f^{\prime}\right) d^{\times} a \\
& =\int_{a \in \varpi^{n} \Theta_{E}^{\times} / E^{1}} \int_{\left(N^{\prime} \times N^{\prime}\right)(F)} f_{1}^{\prime}\left(\hat{n}_{1}^{-1} \gamma_{a} \hat{n}_{2}\right) \psi^{\prime}\left(\hat{n}_{1} \hat{n}_{2}^{-1}\right) d \hat{n}_{1} \\
& \quad \times \int_{\left(N^{\prime} \times N^{\prime}\right)(F)} f_{2}^{\prime}\left(n_{1}^{-1} \gamma_{a} n_{2}\right) \psi^{\prime}\left(n_{1}^{-1} n_{2}\right) u\left(\hat{n}_{2}^{-1} n_{2}, 2 c\right) d n_{2} d n_{1} d \hat{n}_{2} d^{\times} a \\
& =\int_{a \in \varpi^{n} \mathscr{O}_{E}^{\times} / E^{1}} \int_{\left(N^{\prime} \times N^{\prime}\right)(F)} f_{1}^{\prime}\left(\hat{n}_{1}^{-1} \gamma_{a} \hat{n}_{2}\right) \psi^{\prime}\left(\hat{n}_{1} \hat{n}_{2}^{-1}\right) d \hat{n}_{2} d \hat{n}_{1} \\
& \times \int_{\left(N^{\prime} \times N^{\prime}\right)(F)} f_{2}^{\prime}\left(n_{1}^{-1} \gamma_{a} n_{2}\right) \psi^{\prime}\left(n_{1}^{-1} n_{2}\right) d n_{2} d n_{1} d^{\times} a .
\end{aligned}
$$

We have shown that the truncated local Kuznetsov trace formula stabilizes. 
Proposition 3.4. For any $f^{\prime}=f_{1}^{\prime} \otimes f_{2}^{\prime} \in C_{c}^{\infty}\left(\widetilde{G}^{\prime}(F) \times \widetilde{G}^{\prime}(F)\right)$ and $t \gg 0$,

$$
\begin{aligned}
\int_{N^{\prime}(F)} \int_{N^{\prime}(F)} K_{f^{\prime}}\left(n_{1}, n_{2}\right) \psi^{\prime}\left(n_{1}^{-1} n_{2}\right) & u\left(n_{1}, t\right) u\left(n_{2}, t\right) d n_{1} d n_{2} \\
= & \int_{a \in E^{\times} / E^{1}} O^{\prime}\left(f_{1}^{\prime}, \psi^{\prime}, a\right) O^{\prime}\left(f_{2}^{\prime}, \bar{\psi}^{\prime}, a\right)|a|_{E} d^{\times} a .
\end{aligned}
$$

3B. The spectral expansion. Now we derive a spectral expansion for the local Kuznetsov trace formula,

$$
\lim _{t \rightarrow \infty} \int_{N^{\prime}(F)} \int_{N^{\prime}(F)} K_{f^{\prime}}\left(n_{1}, n_{2}\right) \psi^{\prime}\left(n_{1}^{-1} n_{2}\right) u\left(n_{1}, t\right) u\left(n_{2}, t\right) d n_{1} d n_{2} .
$$

Our main tool is the Plancherel formula for $p$-adic groups, which was first stated, with an outlined proof, by Harish-Chandra [1984]. Silberger [1996] later filled in an important proof of one of the steps in the theorem. More recently Waldspurger [2003] provided a complete proof.

As in [Arthur 1991, §2], we begin by rewriting $K_{f^{\prime}}(x, y)$ using the Plancherel formula. First we introduce some additional notation. For an irreducible representation $\left(\sigma, V_{\sigma}\right)$ of $G^{\prime}(F)$ let $\mathscr{B}(\sigma)$ be the Hilbert space of Hilbert-Schmidt operators on $V_{\sigma}$. The inner product on $\mathscr{B}(\sigma)$ is defined as

$$
\left\langle S, S^{\prime}\right\rangle:=\operatorname{tr}\left(S S^{*}\right)
$$

for $S, S^{\prime} \in \mathscr{B}(\sigma)$, where $\operatorname{tr}\left(S S^{* *}\right)=\sum_{\text {o.n.b. } V_{\sigma}}\left\langle S S^{\prime *} u_{i}, u_{i}\right\rangle$ and this sum converges absolutely and does not depend on the basis. For a discrete series representation $\sigma$ of a group $G$ let $d(\sigma)$ be the formal degree of $\sigma$.

Let $\Pi_{2}\left(\widetilde{G}^{\prime}(F)\right)$ be a set of representatives for the equivalence classes of irreducible, tempered square integrable representations of $\widetilde{G}^{\prime}(F)$ and let $\left\{\Pi_{2}\left(\tilde{M}^{\prime}(F)\right)\right\}$ be a set of representatives of unitary characters $\chi$ on $\tilde{M}^{\prime}(F)$ such that $\chi(\varpi)=1$. For a character $\chi$ of $M^{\prime}(F)$ and $\lambda \in \mathbb{C}$, let $\chi_{\lambda}(m)=\chi(m) e^{\lambda\left(H_{P^{\prime}}(m)\right)}$. For $\chi \in\left\{\Pi_{2}\left(\tilde{M}^{\prime}(F)\right)\right\}$, $I_{P^{\prime}}^{G^{\prime}}\left(\chi_{\lambda}\right)=I_{P^{\prime}}\left(\chi_{\lambda}\right)$ is the normalized induced representation of $\widetilde{G}^{\prime}(F)$ acting on a Hilbert space $\mathscr{H}_{P^{\prime}}(\chi)$ of vector-valued functions on $K^{\prime}$. Let $\mathscr{B}_{P^{\prime}}(\chi)$ be a fixed $K^{\prime}$-finite orthonormal basis of the Hilbert space of Hilbert-Schmidt operators on $\mathscr{H}_{P^{\prime}}(\chi)$.

Let $m(\sigma)$ be the Plancherel density. We normalize our measures following [Arthur 1991, §1]. The Plancherel density satisfies $m\left(\chi_{\lambda}\right)=d(\chi) \mu\left(\chi_{\lambda}\right)$, where $\mu\left(\chi_{\lambda}\right)$ is Harish-Chandra's $\mu$-function.

For a fixed $x \in G^{\prime}(F)$, let

$$
h(v)=\int_{\widetilde{G}^{\prime}(F)} f_{1}^{\prime}(x u) f_{2}^{\prime}(u v x) d u .
$$


Then $h \in C_{c}^{\infty}\left(\widetilde{G}^{\prime}(F)\right)$ and $K_{f^{\prime}}(x, y)=h\left(y x^{-1}\right)$, so by the Plancherel formula,

$$
\begin{aligned}
K_{f^{\prime}}(x, y)=\sum_{\sigma \in \Pi_{2}\left(\widetilde{G}^{\prime}(F)\right)} d(\sigma) & \operatorname{tr}\left(\sigma\left(R\left(y x^{-1}\right) h\right)\right) \\
& +\frac{1}{2} \sum_{\chi \in\left\{\Pi_{2}\left(\widetilde{M}^{\prime}(F)\right)\right\}} \int_{0}^{\frac{\pi i}{\log q}} \operatorname{tr}\left(I_{P^{\prime}}\left(\chi, R\left(y x^{-1}\right) h\right)\right) m\left(\chi_{\lambda}\right) d \lambda .
\end{aligned}
$$

Because $I_{P^{\prime}}\left(\chi_{\lambda}, R\left(y x^{-1}\right) h\right) \stackrel{\chi \in\left\{\Pi_{2}\left(\widetilde{M}^{\prime}(F)\right)\right\}}{=} I_{P^{\prime}}\left(\chi_{\lambda}, f_{1}^{\prime \vee}\right) I_{P^{\prime}}\left(\chi_{\lambda}, x\right) I_{P^{\prime}}\left(\chi_{\lambda}, f_{2}^{\prime}\right)\left(I_{P^{\prime}}\left(\chi_{\lambda}, y\right)\right)^{*}$, we have

$$
\begin{aligned}
\operatorname{tr}\left(I_{P^{\prime}}\left(\chi_{\lambda}, R\left(y x^{-1}\right) h\right)\right) & =\sum_{S \in \mathscr{P}_{P^{\prime}}(\chi)}\left(I_{P^{\prime}}\left(\chi_{\lambda}, f_{1}^{\prime \vee}\right) I_{P^{\prime}}\left(\chi_{\lambda}, x\right) I_{P^{\prime}}\left(\chi_{\lambda}, f_{2}^{\prime}\right), S^{*}\right) \overline{\left(I_{P^{\prime}}\left(\chi_{\lambda}, y\right), S^{*}\right)} \\
& =\sum_{S \in \mathscr{P}_{P^{\prime}}(\chi)} \operatorname{tr}\left(I_{P^{\prime}}\left(\chi_{\lambda}, f_{1}^{\prime \vee}\right) I_{P^{\prime}}\left(\chi_{\lambda}, x\right) I_{P^{\prime}}\left(\chi_{\lambda}, f_{2}^{\prime}\right) S\right) \overline{\operatorname{tr}\left(I_{P^{\prime}}\left(\chi_{\lambda}, y\right) S\right)} \\
& =\sum_{S \in \mathscr{P}_{P^{\prime}}(\chi)} \operatorname{tr}\left(I_{P^{\prime}}\left(\chi_{\lambda}, x\right) S_{\lambda}\left[f^{\prime}\right] \overline{\operatorname{tr}\left(I_{P^{\prime}}\left(\chi_{\lambda}, y\right) S\right)},\right.
\end{aligned}
$$

where $S_{\lambda}\left[f^{\prime}\right]=I_{P^{\prime}}\left(\chi_{\lambda}, f_{2}^{\prime}\right) S I_{P^{\prime}}\left(\chi_{\lambda}, f_{1}^{\prime \vee}\right)$.

For $f^{\prime} \in C_{c}^{\infty}\left(\widetilde{G}^{\prime}(F)\right), \pi$ an admissible representation, $\pi\left(f^{\prime}\right)$ has finite rank. Thus the sum over $S$ is a finite sum of an orthonormal basis of operators on $\mathscr{H}_{P}(\chi)^{K_{0}}$ for some open compact $K_{0}$.

Putting everything together we have

$$
\begin{aligned}
& \int_{N^{\prime}(F)} \int_{N^{\prime}(F)} K_{f_{1}^{\prime} \otimes f_{2}^{\prime}}\left(n_{1}, n_{2}\right) \psi^{\prime}\left(n_{1}^{-1} n_{2}\right) u\left(n_{1}, t\right) u\left(n_{2}, t\right) d n_{1} d n_{2} \\
& =\sum_{\sigma \in \Pi_{2}\left(\widetilde{G}^{\prime}(F)\right)} d(\sigma) \sum_{S \in \mathscr{B}^{(}(\sigma)}\left(\int_{N^{\prime}(F)} \operatorname{tr}\left(\sigma(n)\left(\sigma\left(f_{2}^{\prime}\right) S \sigma\left(f_{1}^{\prime \vee}\right)\right)\right) \psi^{\prime}\left(n^{-1}\right) u(n, t) d n\right. \\
& \quad \times \int_{N^{\prime}(F)}^{\left.\operatorname{tr}(\sigma(n) S) \psi^{\prime}\left(n^{-1}\right) u(n, t) d n\right)} \\
& +\frac{1}{2} \sum_{\chi \in\left\{\Pi_{2}\left(\widetilde{M}^{\prime}(F)\right)\right\}} d(\chi) \times \int_{0}^{\frac{\pi i}{\log q}}\left(\sum_{S \in \mathscr{P}_{P^{\prime}}(\chi)} \int_{N^{\prime}(F)} \operatorname{tr}\left(I_{P^{\prime}}\left(\chi_{\lambda}, n\right) S_{\lambda}\left[f^{\prime}\right]\right) \psi^{\prime}\left(n^{-1}\right) u(n, t) d n\right.
\end{aligned}
$$$$
\left.\times \int_{N^{\prime}(F)} \operatorname{tr}\left(I_{P^{\prime}}\left(\chi_{\lambda}, n\right) S\right) \psi^{\prime}\left(n^{-1}\right) u(n, t) d n\right) \mu\left(\chi_{\lambda}\right) d \lambda .
$$

To finish the spectral expansion we show that the above unipotent integrals stabilize. We first note that in the discrete series case the above integrals are absolutely convergent without any truncation for reasons similar to those in Section 4B1.

Lemma 3.5 (spectral stabilization). For any complex-valued function $\phi$ on $\widetilde{G}^{\prime}(F)$ that is biinvariant under a fixed open compact subgroup, there exists a positive 
integer $c$ such that for all $t \geq c$,

$$
\int_{N^{\prime}(F)} \phi(n) \psi^{\prime}(n) u(n, t) d n=\int_{N^{\prime}(F)} \phi(n) \psi^{\prime}(n) u(n, c) d n .
$$

This $c$ only depends on the open compact subgroup under which $\phi$ is biinvariant.

Proof. Let $K_{1}$ be an open compact subgroup of $\widetilde{G}^{\prime}(F)$ under which $\phi$ is biinvariant. $K_{1}$ must contain a neighborhood of the identity, so there exists a positive integer $c^{\prime}$ such that

$$
\left(\begin{array}{cc}
a & 0 \\
0 & \bar{a}^{-1}
\end{array}\right) \in K_{1} \quad \text { for all } a \in\left(1+\varpi^{c^{\prime}} \mathcal{O}_{E}\right) E^{1}
$$

We show that for $m>c^{\prime}$,

$$
\int_{\varpi^{-m} \mathbb{O}_{F}^{\times}} \phi\left(\left(\begin{array}{ll}
1 & x \\
0 & 1
\end{array}\right)\right) \psi^{\prime}(x) d x=0 .
$$

We note that

$$
\left(\begin{array}{cc}
a & 0 \\
0 & \bar{a}^{-1}
\end{array}\right)\left(\begin{array}{cc}
1 & x \\
0 & 1
\end{array}\right)\left(\begin{array}{cc}
a^{-1} & 0 \\
0 & \bar{a}
\end{array}\right)=\left(\begin{array}{cc}
1 & a \bar{a} x \\
0 & 1
\end{array}\right)
$$

Thus for $x^{\prime} \in 1+\varpi^{c^{\prime}} 0_{F}$,

$$
\phi\left(\left(\begin{array}{cc}
1 & x^{\prime} x \\
0 & 1
\end{array}\right)\right)=\phi\left(\left(\begin{array}{ll}
1 & x \\
0 & 1
\end{array}\right)\right) .
$$

Hence

$$
\begin{aligned}
\int_{\varpi^{-m} \mathscr{O}_{F}^{\times}} \phi\left(\left(\begin{array}{ll}
1 & x \\
0 & 1
\end{array}\right)\right) \psi^{\prime}(x) d x & \\
= & \sum_{\alpha \in \mathbb{O}_{F}^{\times} /\left(1+\varpi^{\left.c^{\prime} \mathscr{O}_{F}\right)}\right.} \int_{\varpi^{-m}\left(1+\varpi^{\left.c^{\prime} \mathscr{O}_{F}\right)}\right.} \phi\left(\left(\begin{array}{cc}
1 & \alpha x \\
0 & 1
\end{array}\right)\right) \psi^{\prime}(\alpha x) d x \\
= & \sum_{\alpha \in \mathbb{O}_{F}^{\times} /\left(1+\varpi^{\left.c^{\prime} \mathscr{O}_{F}\right)}\right.} \phi\left(\left(\begin{array}{cc}
1 & \varpi^{-m} \alpha \\
0 & 1
\end{array}\right)\right) \psi^{\prime}\left(\varpi^{-m} \alpha\right) \int_{\varpi^{c^{\prime}-m \mathscr{O}_{F}}} \psi^{\prime}(x) d x .
\end{aligned}
$$

The last line equals 0 for $m>c^{\prime}$. Thus for $t>2 c^{\prime}$,

$$
\int_{N^{\prime}(F)} \phi(n) \psi^{\prime}(n) u(n, t) d n=\int_{N^{\prime}(F)} \phi(n) \psi^{\prime}(n) u\left(n, 2 c^{\prime}\right) d n .
$$

We have now proved the following. 
Proposition 3.6. For any $f^{\prime}=f_{1}^{\prime} \otimes f_{2}^{\prime} \in C_{c}^{\infty}\left(\widetilde{G}^{\prime}(F) \times \widetilde{G}^{\prime}(F)\right)$, $\lim _{t \rightarrow \infty} \int_{N^{\prime}(F)} \int_{N^{\prime}(F)} K_{f^{\prime}}\left(n_{1}, n_{2}\right) \psi^{\prime}\left(n_{1}^{-1} n_{2}\right) u\left(n_{1}, t\right) u\left(n_{2}, t\right) d n_{1} d n_{2}$ where

$$
=\sum_{\sigma^{\prime} \in \Pi_{2}\left(\widetilde{G}^{\prime}(F)\right)} d\left(\sigma^{\prime}\right) D_{\sigma^{\prime}}^{\prime}\left(f^{\prime}\right)+\frac{1}{2} \sum_{\chi^{\prime} \in\left\{\Pi_{2}\left(\widetilde{M}^{\prime}(F)\right)\right\}} d\left(\chi^{\prime}\right) \int_{0}^{\frac{\pi i}{\log q}} D_{\chi_{\lambda}^{\prime}}^{\prime}\left(f^{\prime}\right) \mu\left(\chi_{\lambda}^{\prime}\right) d \lambda,
$$

$$
\begin{aligned}
D_{\sigma^{\prime}}^{\prime}\left(f^{\prime}\right) & =\sum_{S \in \mathscr{B}\left(\sigma^{\prime}\right)} W_{\sigma^{\prime}}^{\prime}\left(\sigma^{\prime}\left(f_{2}^{\prime}\right) S \sigma^{\prime}\left(f_{1}^{\prime \vee}\right)\right) \overline{W_{\sigma^{\prime}}^{\prime}(S)}, \\
W_{\sigma^{\prime}}^{\prime}(S) & =\int_{N^{\prime}(F)} \operatorname{tr}\left(\sigma^{\prime}(n) S\right) \psi^{\prime}\left(n^{-1}\right) d n \\
D_{\chi_{\lambda}^{\prime}}^{\prime}\left(f^{\prime}\right) & =\sum_{S \in \mathscr{P}_{P^{\prime}}\left(\chi^{\prime}\right)} W_{\chi_{\lambda}^{\prime}}^{\prime}\left(I_{P^{\prime}}\left(\chi_{\lambda}^{\prime}, f_{2}^{\prime}\right) S I_{P^{\prime}}\left(\chi_{\lambda}^{\prime}, f_{1}^{\prime \vee}\right)\right) \overline{W_{\chi_{\lambda}^{\prime}}^{\prime}(S)}, \\
W_{\chi_{\lambda}^{\prime}}^{\prime}(S) & =\lim _{t \rightarrow \infty} \int_{N^{\prime}(F)} \operatorname{tr}\left(I_{P^{\prime}}\left(\chi_{\lambda}^{\prime}, n\right) S\right) \psi^{\prime}\left(n^{-1}\right) u(n, t) d n .
\end{aligned}
$$

We note that Theorem 1.3 now follows from the results of Propositions 3.4 and 3.6.

\section{The local relative trace formula and periods for PGL(2)}

In this section we define a local relative trace formula for PGL(2). We expand this local relative trace formula geometrically in terms of separate orbital integrals of $f_{1}$ and $f_{2}$. Then we use Harish-Chandra's Plancherel formula to rewrite this expression spectrally in terms of representations. We define a regularized period integral, show that it is an $\mathrm{H} \times \mathrm{H}$-invariant linear functional and that it is the term that appears in the spectral expansion of the local relative trace formula.

We define the local relative trace formula for $f=f_{1} \otimes f_{2} \in C_{c}^{\infty}(\widetilde{G}(F) \times \widetilde{G}(F))$ as the equality between the geometric and spectral expansions of

$$
\lim _{t \rightarrow \infty} \int_{\widetilde{H}(F)} \int_{N(F)} K_{f}(h, n) \psi(n) u(h, t) u(n, t) d n d h
$$

where

$$
K_{f}(h, n)=\int_{\widetilde{G}(F)} f_{1}(g) f_{2}\left(h^{-1} g n\right) d g .
$$

As we did with the local Kuznetsov trace formula, we show that for a fixed $f$ this limit stabilizes.

4A. The geometric expansion. We will rewrite

$$
\lim _{t \rightarrow \infty} \int_{\widetilde{H}(F)} \int_{N(F)} K_{f}(h, n) \psi(n) u(h, t) u(n, t) d n d h
$$


as an integral over admissible cosets of a product of an orbital integral for $f_{1}$ and an orbital integral $f_{2}$.

4A1. Integration formula. As pointed out in [Jacquet et al. 1999, §VI.13], by [Springer 1985], $G(F)=H(F) P(F) \sqcup H(F) \eta P(F)$, where $\eta$ is any element in $G(F)$ such that $\bar{\eta}^{-1} \eta=\left(\begin{array}{ll}0 & 1 \\ 1 & 0\end{array}\right)$. Let $\eta_{a}=\eta\left(\begin{array}{ll}a & 0 \\ 0 & 1\end{array}\right)$ and $\gamma_{\alpha}=\left(\begin{array}{cc}1 & 0 \\ 0 & \alpha+\sqrt{\tau}\end{array}\right)$, where $E=F(\sqrt{\tau})$. Then

$\left\{\left(\begin{array}{ll}1 & 0 \\ 0 & 1\end{array}\right)\right\} \cup\left\{\gamma_{\alpha}: \alpha \in F\right\} \cup\left\{\eta_{a}: a\right.$ is in a set of representatives for $\left.E^{\times} / E^{1}\right\}$ is a set of representatives for the double cosets of $\widetilde{H}(F) \backslash \widetilde{G}(F) / N(F)$.

For $g \in G(F)$, let $C_{g}(\widetilde{H}(F) \times N(F))=\left\{(h, n) \in \widetilde{H}(F) \times N(F): h^{-1} g n=z g\right.$ for some $\left.z \in Z(F)\right\}$.

Definition 4.1. An element $g \in \widetilde{G}(F)$ and its corresponding orbit is called admissible if the map $C_{g}(\widetilde{H}(F) \times N(F)) \rightarrow \mathbb{C}:(h, n) \mapsto \psi(n)$ is trivial.

By a short calculation we see that

$$
\begin{aligned}
& C_{\gamma_{\alpha}}(\tilde{H}(F) \times N(F))=\left\{\left(\left(\begin{array}{ll}
1 & y \\
0 & 1
\end{array}\right),\left(\begin{array}{cc}
1 & y(\alpha+\sqrt{\tau}) \\
0 & 1
\end{array}\right)\right): y \in F\right\}, \\
& C_{\eta_{a}}(\tilde{H}(F) \times N(F))=1 .
\end{aligned}
$$

Thus the orbits represented by $\left\{\eta_{a}: a \in E^{\times} / E^{1}\right\} \cup\left\{\gamma_{0}\right\}$ are admissible.

We have the following integration formula. For any $F \in C_{c}(\widetilde{G}(F))$,

$$
\int_{\widetilde{G}(F)} F(g) d g=\int_{E^{\times} / E^{1}} \int_{\widetilde{H}(F) \times N(F)} F\left(h^{-1} \eta_{a} n\right) d n d h|a|_{E} d^{\times} a .
$$

4A2. Separating the orbital integrals. Let

$$
R^{t}(f)=\int_{\widetilde{H}(F)} \int_{N(F)} K_{f}(h, n) \psi(n) u(h, t) u(n, t) d n d h .
$$

$R^{t}(f)$ is absolutely convergent because $f_{1}(g), u(h, t)$ and $u(n, t)$ have compact support on $\widetilde{G}(F), \widetilde{H}(F)$ and $N(F)$ respectively. By changing the order of integration and applying (4-1) we see that $R^{t}(f)$ equals

$$
\begin{aligned}
\int_{E^{\times} / E^{1}} \int_{\widetilde{H}(F) \times N(F)} \int_{\widetilde{H}(F) \times N(F)} f_{1}\left(h_{1}^{-1} \eta_{a} n_{1}\right) f_{2}\left(h_{2}^{-1} h_{1}^{-1} \eta_{a} n_{1} n_{2}\right) \\
\times \psi\left(n_{2}\right) u\left(h_{2}, t\right) u\left(n_{2}, t\right) d n_{2} d h_{2} d n_{1} d h_{1}|a|_{E} d^{\times} a .
\end{aligned}
$$

By a change of variables we have

$$
R^{t}(f)=\int_{E^{\times} / E^{1}} R^{t}\left(\eta_{a}, f\right)|a|_{E} d^{\times} a,
$$


where

$$
\begin{array}{r}
R^{t}\left(\eta_{a}, f\right)=\int_{\widetilde{H}(F) \times N(F)} \int_{\widetilde{H}(F) \times N(F)} f_{1}\left(h_{1}^{-1} \eta_{a} n_{1}\right) f_{2}\left(h_{2}^{-1} \eta_{a} n_{2}\right) \psi\left(n_{1}^{-1} n_{2}\right) \\
\times u\left(h_{1}^{-1} h_{2}, t\right) u\left(n_{1}^{-1} n_{2}, t\right) d n_{2} d h_{2} d n_{1} d h_{1} .
\end{array}
$$

To complete the geometric expansion of the local relative trace formula we rewrite $R^{t}(f)$ for $t \gg 0$ as an integral of a product of two separate orbital integrals that are not truncated. We omit the proof the lemma below as it is very similar to the proof of Lemma 3.3.

Lemma 4.2. For $f \in C_{c}^{\infty}(\widetilde{G}(F) \times \widetilde{G}(F))$, there exists a $T>0$ such that for all $t \geq T$ and $n \in \mathbb{Z}$,

$$
\int_{\varpi^{n} \mathscr{O}_{E}^{\times} / E^{1}} R^{t}\left(\eta_{a}, f\right) d^{\times} a=\int_{\varpi^{n} \mathscr{O}_{E}^{\times} / E^{1}} O\left(f_{1}, \psi, a\right) O\left(f_{2}, \bar{\psi}, a\right) d^{\times} a
$$

where

$$
O(f, \psi, a)=\int_{\widetilde{H}(F)} \int_{N(F)} f\left(h^{-1} \eta_{a} n\right) \overline{\psi(n)} d n d h .
$$

We have proved the following proposition.

Proposition 4.3. For any $f=f_{1} \otimes f_{2} \in C_{c}^{\infty}(\widetilde{G}(F) \times \widetilde{G}(F))$, $\lim _{t \rightarrow \infty} \int_{\widetilde{H}(F)} \int_{N(F)} K(h, n) \psi(n) u(h, t) u(n, t) d n d h$

$$
=\int_{a \in E^{\times} / E^{1}} O\left(f_{1}, \psi, a\right) O\left(f_{2}, \bar{\psi}, a\right)|a|_{E} d^{\times} a .
$$

Here, as in the local Kuznetsov trace formula, we have actually shown that the limit of the truncated local relative trace formula stabilizes.

4B. The spectral expansion and period integrals. We want to develop a spectral expansion for the local relative trace formula,

$$
\lim _{t \rightarrow \infty} \int_{\widetilde{H}(F)} \int_{N(F)} K_{f}(h, n) \psi(n) u(h, t) u(n, t) d n d h .
$$

As in the previous section, we expand the kernel via the Plancherel formula:

$$
\begin{aligned}
\int_{\widetilde{H}(F)} & \int_{N(F)} K(h, n) \psi(n) u(h, t) u(n, t) d n d h \\
\quad= & \sum_{\sigma \in \Pi_{2}(\widetilde{G}(F))} d(\sigma) D_{\sigma}^{t}(f)+\frac{1}{2} \sum_{\chi \in\left\{\Pi_{2}(\widetilde{M}(F))\right\}} d(\chi) \int_{0}^{\frac{\pi i}{\log q}} \mu\left(\chi_{\lambda}\right) D_{\chi_{\lambda}}^{t}(f) d \lambda
\end{aligned}
$$


where

$$
\begin{aligned}
D_{\sigma}^{t}(f) & =\sum_{S \in \mathscr{B}(\sigma)} P_{\sigma}^{t}\left(\sigma\left(f_{2}\right) S \sigma\left(f_{1}^{\vee}\right)\right) \overline{W_{\sigma}^{t}(S)}, \\
D_{\chi_{\lambda}}^{t}(f) & =\sum_{S \in \mathscr{B}_{P}(\chi)} P_{I_{P}\left(\chi_{\lambda}\right)}^{t}\left(I_{P}\left(\chi_{\lambda}, f_{2}\right) S I_{P}\left(\chi_{\lambda}, f_{1}^{\vee}\right)\right) \overline{W_{I_{P}\left(\chi_{\lambda}\right)}^{t}(S)}, \\
W_{\pi}^{t}(S) & =\int_{N(F)} \operatorname{tr}(\pi(n) S) \psi\left(n^{-1}\right) u(n, t) d n, \\
P_{\pi}^{t}(S) & =\int_{\widetilde{H}(F)} \operatorname{tr}(\pi(h) S) u(h, t) d h .
\end{aligned}
$$

By Lemma 3.5, there exists a positive integer $c$, such that for $t>c$,

$$
W_{\pi}^{t}(S)=\int_{N(F)} \operatorname{tr}(\pi(n) S) \psi\left(n^{-1}\right) u(n, c) d n .
$$

Thus as in the previous section, we define

$$
\begin{aligned}
W_{\sigma}(S) & =\int_{N(F)} \operatorname{tr}(\sigma(n) S) \psi\left(n^{-1}\right) d n, \\
W_{\chi_{\lambda}}(S) & =\lim _{t \rightarrow \infty} \int_{N(F)} \operatorname{tr}\left(I_{P}\left(\chi_{\lambda}, n\right) S\right) \psi\left(n^{-1}\right) u(n, t) d n .
\end{aligned}
$$

To finish the spectral expansion of the local relative trace formula we need to define the regularized integral

$$
\int_{\widetilde{H}(F)}^{*} \operatorname{tr}\left(I_{P}\left(\chi_{\lambda}, h\right) S\right) d h
$$

because $\operatorname{tr}\left(I_{P}\left(\chi_{\lambda},-\right) S\right)$ is not integrable over $\widetilde{H}(F)$.

Many of the techniques in this section are inspired by the work of Jacquet, Lapid and Rogawski in [Jacquet et al. 1999]. In that paper they define a regularized period integral for an automorphic form $\phi$ on $G(\mathbb{A})$ integrated over $H$ where $G$ is a reductive group over a number field $F$ and $H$ is the fixed point set of an involution of $G$. They focus on the case $G=\operatorname{Res}_{E / F} H$ where $E / F$ is a quadratic extension and they obtain explicit results for $G=\mathrm{GL}(n, E), H=\mathrm{GL}(n, F)$.

For $\lambda \in \mathbb{C}$ and $m=\left(\begin{array}{ll}\alpha & 0 \\ 0 & \beta\end{array}\right) \in M(F)$, let $e^{\lambda H_{M}(m)}=|\alpha / \beta|_{E}^{\lambda}$. If $g=m(g) n(g) k(g)$, $m(g) \in M(F), n(g) \in N(F), k(g) \in K$, we let $e^{\lambda H_{P}(g)}=e^{\lambda H_{M}(m(g))}$. Let $\delta_{P}(m)=$ $e^{H_{M}(m)}$. We give analogous definitions for $e^{\lambda H_{M_{H}}}$ and $\delta_{P_{H}}$ so that for $m \in M_{H}(F)$, $e^{\lambda H_{M}(m)}=e^{2 \lambda H_{M_{H}}(m)}$.

We recall the Cartan decomposition $H(F)=K_{H} M_{H}^{+}(F) K_{H}$, where

$$
M_{H}^{+}(F)=\left\{\left(\begin{array}{ll}
\alpha & 0 \\
0 & \beta
\end{array}\right) \in M(F): v\left(\frac{\alpha}{\beta}\right) \leq 0\right\} .
$$


Then for any absolutely integrable function $f$

$$
\int_{\widetilde{H}(F)} f(h) d h=\int_{\widetilde{K}_{H}} \int_{\widetilde{K}_{H}} \int_{\widetilde{M}_{H}^{+}(F)} D_{P_{H}}(m) f\left(k_{1} m k_{2}\right) d m d k_{2} d k_{1},
$$

where

$$
D_{P_{H}}\left(\left(\begin{array}{ll}
\alpha & 0 \\
0 & \beta
\end{array}\right)\right)= \begin{cases}|\alpha / \beta|_{F}\left(1+|\varpi|_{F}\right) & v(\alpha / \beta) \leq 0 \\
0 & v(\alpha / \beta)>0\end{cases}
$$

To define the regularized integral, we begin by defining a regularized integral on $M_{H}^{+}(F)$. We note that

$$
1-u\left(\left(\begin{array}{rr}
1 & \\
& \alpha
\end{array}\right), t\right)= \begin{cases}0 & 0 \leq v(\alpha) \leq t \\
1 & v(\alpha)>t\end{cases}
$$

For $\operatorname{Re} v<-\operatorname{Re} \lambda$,

$$
\int_{\widetilde{M}_{H}^{+}(F)} e^{(\nu+\lambda) H_{M}(m)}(1-u(m, t)) d m=\sum_{n=t+1}^{\infty} q^{2 n(\nu+\lambda)}=\frac{q^{(t+1) 2(\nu+\lambda)}}{1-q^{2(\nu+\lambda)}} .
$$

We write

$$
\int_{\widetilde{M}_{H}^{+}(F)}^{\sharp} e^{\lambda H_{M}(m)}(1-u(m, t)) d m
$$

to denote the meromorphic continuation at $v=0$ of (4-3). This is well-defined so long as $\lambda \neq 0$. Let

$$
\begin{gathered}
\phi\left(k_{1} m k_{2}\right)=\sum_{i=1}^{r} \phi_{i}\left(k_{1}, k_{2}\right) f_{i}(m) e^{\lambda_{i} H_{M}(m)}, \quad k_{1}, k_{2} \in K_{H}, \\
m=\left(\begin{array}{cc}
1 & \\
& \varpi^{n}
\end{array}\right), \quad n \geq 0, \quad f_{i} \in C_{c}(\tilde{M}(F))
\end{gathered}
$$

with $\lambda_{i} \neq-\frac{1}{2}$. We define for $t \gg 0$,

$$
\begin{aligned}
\int_{\widetilde{H}(F)}^{\sharp} \phi & (h)(1-u(h, t)) d h \\
= & \sum_{i=1}^{r} \int_{\widetilde{K}_{H} \times \widetilde{K}_{H}} \phi_{i}\left(k_{1}, k_{2}\right) \int_{\widetilde{M}_{H}^{+}(F)}^{\sharp} D_{P_{H}}(m) e^{\lambda_{i} H_{M}(m)}(1-u(m, t)) d m \\
& =\left(1+q^{-1}\right) \sum_{i=1}^{r} \int_{\widetilde{K}_{H} \times \widetilde{K}_{H}} \phi_{i}\left(k_{1}, k_{2}\right) \int_{\widetilde{M}_{H}^{+}(F)}^{\sharp} e^{\left(\lambda_{i}+1 / 2\right) H_{M}(m)}(1-u(m, t)) d m .
\end{aligned}
$$

If $\phi$ is a matrix coefficient of $I_{P}\left(\chi_{\lambda}\right)$ where $\chi(\varpi)=1$ then by smoothness and the asymptotics of matrix coefficients there exists a function $C^{P} \phi$ of the form in 
(4-4) with $\lambda_{i} \in\left\{\lambda-\frac{1}{2},-\lambda-\frac{1}{2}\right\}$ and for $n \gg 0$,

$$
C^{P} \phi\left(k_{1}\left(\begin{array}{cc}
1 & \\
& \varpi^{n}
\end{array}\right) k_{2}\right)=\phi\left(k_{1}\left(\begin{array}{ll}
1 & \\
& \varpi^{n}
\end{array}\right) k_{2}\right) .
$$

Note that the condition for the regularized integral to exist is now that $\lambda \neq 0$.

Definition 4.4. For any matrix coefficient $\phi$ of $I_{P}\left(\chi_{\lambda}\right)$ such that $\chi(\varpi)=1$ and $\lambda \neq 0$,

$$
\int_{\widetilde{H}(F)}^{*} \phi(h) d h:=\int_{\widetilde{H}(F)} \phi(h) u(h, t) d h+\int_{\widetilde{H}(F)}^{\sharp} \phi(h)(1-u(h, t)) d h
$$

for $t \gg 0$.

One can check that this definition of the regularized integral is independent of $t$ and agrees with the usual integral if we start with something that is integrable. Now we will prove that it is $H$-invariant and then we will explicitly relate the regularized period to the truncated period that occurs in the local trace formula.

Let $\phi^{h_{0}}(x)=\phi\left(x h_{0}\right)$ for $h_{0} \in \widetilde{H}$. Note that if $\phi$ is a matrix coefficient of $\pi$ then $\phi^{h_{0}}$ is as well.

Lemma 4.5. Fix $h_{0} \in H, \lambda \neq 0$ and a character $\chi$ of $E^{\times}$with $\chi(\varpi)=1$. Then for any matrix coefficient $\phi$ of $I_{P}\left(\chi_{\lambda}\right)$ and $t \gg 0$,

$$
\begin{aligned}
\int_{\widetilde{K}_{H} \times \widetilde{K}_{H}} \int_{\widetilde{M}_{H}(F)}^{\sharp} D_{P_{H}}(m) \phi^{h_{0}}\left(k_{1} m k_{2}\right)\left(1-u\left(k_{1} m k_{2} h_{0}, t\right)\right) d m d k_{1} d k_{2} \\
=\int_{\widetilde{K}_{H} \times \widetilde{K}_{H}} \int_{\widetilde{M}_{H}(F)}^{\sharp} D_{P_{H}}(m) \phi\left(k_{1} m k_{2}\right)(1-u(m, t)) d m d k_{1} d k_{2} .
\end{aligned}
$$

Proof. For $g \in G(F)$ let $\mathcal{M}(g) \in M^{+}(F)$ be such that $g=k_{1} \mathcal{M}(g) k_{2}, k_{1}, k_{2} \in K$. For $\operatorname{Re} v \ll 0$ and $t \gg 0$,

$$
\begin{aligned}
\int_{\widetilde{K}_{H} \times \widetilde{K}_{H}} \int_{\widetilde{M}_{H}(F)} D_{P_{H}}(m) \phi^{h_{0}}\left(k_{1} m k_{2}\right) \\
\quad \times e^{\nu\left(H_{M}\left(\mathcal{M}\left(k_{1} m k_{2} h_{0}\right)\right)\right)}\left(1-u\left(k_{1} m k_{2} h_{0}, t\right)\right) d m d k_{1} d k_{2} \\
=\int_{\widetilde{H}(F)} \phi\left(h h_{0}\right) e^{\nu\left(H_{M}\left(\mathcal{M}\left(h h_{0}\right)\right)\right)}\left(1-u\left(h h_{0}, t\right)\right) d h \\
=\int_{\widetilde{H}(F)} \phi(h) e^{\nu\left(H_{M}(\mathcal{M}(h))\right)}(1-u(h, t)) d h \\
=\int_{\widetilde{K}_{H} \times \widetilde{K}_{H}} \int_{\widetilde{M}_{H}(F)} D_{P_{H}}(m) \phi\left(k_{1} m k_{2}\right) e^{\nu\left(H_{M}(m)\right)}(1-u(m, t)) d m d k_{1} d k_{2}
\end{aligned}
$$

by the invariance of Haar measure, since both sides are absolutely convergent. For $t \gg 0$, if $h \in \operatorname{supp}(1-u(\cdot, t))$, then $\mathcal{M}\left(h h_{0}\right)=\mathcal{M}(h) \mathcal{M}\left(k_{2} h_{0}\right)$. Thus both sides of the equation above have a meromorphic continuation whose value at $v=0$ gives the statement of the lemma. 
Proposition 4.6 ( $H$-invariance). Let $\phi$ be a matrix coefficient of $I_{P}\left(\chi_{\lambda}\right)$, where $\chi(\varpi)=1$ and $\lambda \neq 0$, and let $h_{0} \in H(F)$. Then

$$
\int_{\widetilde{H}(F)}^{*} \phi^{h_{0}}(h) d h=\int_{\widetilde{H}(F)}^{*} \phi(h) d h .
$$

Proof. By the definition of the regularized integrals, the statement of the proposition will follow once we prove the following equality:

$$
\begin{array}{r}
\int_{\widetilde{K}_{H} \times \widetilde{K}_{H}} \int_{\widetilde{M}_{H}^{+}(F)}^{\sharp} D_{P_{H}}(m) \phi^{h_{0}}\left(k_{1} m k_{2}\right)(1-u(m, t)) d m d k_{1} d k_{2} \\
-\int_{\widetilde{K}_{H} \times \widetilde{K}_{H}} \int_{\widetilde{M}_{H}^{+}(F)}^{\sharp} D_{P_{H}}(m) \phi\left(k_{1} m k_{2}\right)(1-u(m, t)) d m d k_{1} d k_{2} \\
=\int_{\widetilde{H}(F)} \phi(h) u(h, t) d h-\int_{\widetilde{H}(F)} \phi^{h_{0}}(h) u(h, t) d h .
\end{array}
$$

First we note that by Lemma 4.5

$$
\begin{aligned}
\int_{\widetilde{K}_{H} \times \widetilde{K}_{H}} \int_{\widetilde{M}_{H}^{+}(F)}^{\sharp} D_{P_{H}}(m) \phi^{h_{0}}\left(k_{1} m k_{2}\right)(1-u(m, t)) d m d k_{1} d k_{2} \\
-\int_{\widetilde{K}_{H} \times \widetilde{K}_{H}} \int_{\widetilde{M}_{H}^{+}(F)}^{\sharp} D_{P_{H}}(m) \phi\left(k_{1} m k_{2}\right)(1-u(m, t)) d m d k_{1} d k_{2} \\
\left.=\int_{\widetilde{K}_{H} \times \widetilde{K}_{H}} \int_{\widetilde{M}_{H}^{+}(F)}^{\sharp} D_{P_{H}}(m) \phi\left(k_{1} m k_{2}\right)\left(1-u\left(m k_{2} h_{0}^{-1}, t\right)\right)\right) d m d k_{1} d k_{2} \\
-\int_{\widetilde{K}_{H} \times \widetilde{K}_{H}} \int_{\widetilde{M}_{H}^{+}(F)}^{\sharp} D_{P_{H}}(m) \phi\left(k_{1} m k_{2}\right)(1-u(m, t)) d m d k_{1} d k_{2} .
\end{aligned}
$$

For fixed $h_{0}$ and $t$ sufficiently large, $u\left(\cdot h_{0}^{-1}, t\right)-u(\cdot, t)$ has support contained in an annulus. From this fact one can easily check that the previous line is equal to the convergent integral

$$
\begin{aligned}
\int_{\widetilde{K}_{H} \times \widetilde{K}_{H}} \int_{\widetilde{M}_{H}^{+}(F)} D_{P_{H}}(m) \phi & \left(k_{1} m k_{2}\right)\left[u(m, t)-u\left(m k_{2} h_{0}^{-1}, t\right)\right] d m d k_{1} d k_{2} . \\
& =\int_{\widetilde{H}(F)} \phi(h)\left[u(h, t)-u\left(h h_{0}^{-1}, t\right)\right] d h \\
& =\int_{\widetilde{H}(F)} \phi(h) u(h, t) d h-\int_{\widetilde{H}(F)} \phi^{h_{0}}(h) u(h, t) d h .
\end{aligned}
$$

We note that Proposition 4.6 also holds if we replace $\phi^{h_{0}}$ with $\phi\left(h_{0}-\right)$ so our regularized integral is $H \times H$ invariant.

Now we derive an explicit formula relating regularized periods to truncated periods for the matrix coefficients that appear in the trace formula. We begin by recalling 
some definitions of Harish-Chandra's. For $\sigma$ an admissible, tempered representation of $G, \mathscr{A}_{\sigma}(G)$ is the space of functions on $G$ spanned by $K$-finite matrix coefficients of $\sigma, \mathscr{A}_{\text {temp }}(G)$ is the sum of $\mathscr{A}_{\sigma}(G)$ over all admissible tempered representations of $G$ and $\mathscr{A}_{2}(G)$ is the sum of $\mathscr{A}_{\sigma}(G)$ over all unitary, square integrable representations. For $\tau$ a finite dimensional, unitary, two-sided representation of $K$,

$\mathscr{A}_{\sigma}(G, \tau)=\left\{f \in \mathscr{A}_{\sigma}(G) \otimes V_{\tau}: f\left(k_{1} g k_{2}\right)=\tau\left(k_{1}\right) f(g) \tau\left(k_{2}\right), g \in G, k_{1}, k_{2} \in K\right\}$.

Then $\mathscr{A}_{\text {temp }}(G, \tau)$ and $\mathscr{A}_{2}(G, \tau)$ are defined similarly.

Let $\tau_{M}=\left.\tau\right|_{K \cap M}$. By [Harish-Chandra 1984, §3] for $f \in \mathscr{A}_{\sigma}(G, \tau)$ there exists a unique function $C^{P} f \in \mathscr{A}\left(M, \tau_{M}\right)$ such that

$$
\lim _{\left|\frac{\alpha}{\beta}\right|_{E} \rightarrow \infty}\left|\delta_{P}\left(\left(\begin{array}{cc}
\alpha & 0 \\
0 & \beta
\end{array}\right)\right)^{\frac{1}{2}} f\left(\left(\begin{array}{cc}
\alpha & 0 \\
0 & \beta
\end{array}\right)\right)-\left(C^{P} f\right)\left(\left(\begin{array}{cc}
\alpha & 0 \\
0 & \beta
\end{array}\right)\right)\right|=0 .
$$

We call $C^{P} f$ the weak constant term of $f$.

For two parabolics $P_{1}, P_{2}$ with Levi component $M$, let

$$
V_{P_{1} \mid P_{2}}=\left\{v \in V: \tau\left(n_{1}\right) v \tau\left(n_{2}\right)=v, n_{1} \in N_{P_{1}} \cap K, n_{2} \in N_{P_{2}} \cap K\right\}
$$

and let $\tau_{P_{1} \mid P_{2}}$ be the subrepresentation of $\tau_{M}$ on $V_{P_{1} \mid P_{2}}$. For $\Psi \in \mathscr{A}_{2}\left(M, \tau_{P \mid P}\right)$ and $\lambda \in[0, \pi i / \log q]$, the Eisenstein integral $E_{P}(g, \Psi, \lambda) \in \mathscr{A}_{\text {temp }}(G, \tau)$ is defined as

$$
E_{P}(g, \Psi, \lambda)=\int_{K} \tau(k)^{-1} \Psi_{P}(k g) e^{(\lambda+1 / 2)\left(H_{M}(k g)\right)} d k
$$

where $\Psi_{P}$ extends $\Psi$ to $G$ by $\Psi_{P}(n m k)=\Psi(m) \tau(k)$ for $n \in N, m \in M, k \in K$.

The weak constant term of the Eisenstein integral uniquely defines HarishChandra's $c$-functions [1984, §6]. For each element $w$ in the Weyl group $W$ of $\widetilde{G}$, the $c$-function $c_{P \mid P}(w, \lambda)$ is a linear map from $\mathscr{A}_{2}\left(M, \tau_{P \mid P}\right)$ to $\mathscr{A}_{2}\left(M, \tau_{P \mid \bar{P}}\right)$ such that

$$
\left(C^{P} E_{P}\right)(m, \Psi, \lambda)=\left(c_{P \mid P}(1, \lambda) \Psi\right)(m) e^{\lambda H_{M}(m)}+\left(c_{P \mid P}(w, \lambda) \Psi\right)(m) e^{-\lambda H_{M}(m)}
$$

where $w$ is a representative for the nontrivial element in the Weyl group of $\widetilde{G}$. Let $c_{P \mid P}(s, \lambda)_{\chi}$ denote the restriction of $c_{P \mid P}(s, \lambda)$ to $\mathscr{A}_{\chi}\left(M,\left(\tau_{\Gamma}\right)_{P \mid P}\right)$. We have

$$
\mu\left(\chi_{\lambda}\right)^{-1}=c_{P \mid P}(s, \lambda)_{\chi}^{*} c_{P \mid P}(s, \lambda)_{\chi} .
$$

For the rest of this section we let $c(1, \lambda)=c_{P \mid P}(1, \lambda)_{\chi}$ and $c(w, \lambda)=c_{P \mid P}(w, \lambda)_{\chi}$.

We note that the $S$ we consider are actually in $\mathscr{H}_{P}(\chi)^{K_{0}}$ for some open compact $K_{0}$. Harish-Chandra [1976, §7] gives an isomorphism $S \rightarrow \Psi_{S}$ from $\operatorname{End}\left(\mathscr{H}_{P}(\chi)^{K}\right)$ onto $\mathscr{A}_{\chi}\left(M,(\tau)_{P \mid P}\right)$ where $V_{\tau}$ is a particular subspace of $L^{2}(K \times K)$ such that

$$
\operatorname{tr}\left(I_{P}\left(\chi_{\lambda}, k_{1} g k_{2}\right) S\right)=E_{P}\left(g, \Psi_{S}, \lambda\right)_{k_{1}, k_{2}} .
$$


We can now relate the regularized integral to what appears in the local relative trace formula.

Proposition 4.7. For $\chi=\left(\chi, \chi^{-1}\right) \in\left\{\Pi_{2}(\tilde{M}(F)\}, \chi(\varpi)=1, \lambda \neq 0, t \gg 0\right.$,

$$
\begin{aligned}
& \int_{\widetilde{H}(F)}^{*} \operatorname{tr}\left(I_{P}\left(\chi_{\lambda}, h\right) S\right) d h \\
& =\int_{\widetilde{H}(F)} \operatorname{tr}\left(I_{P}\left(\chi_{\lambda}, h\right) S\right) u(h, t) d h \\
& \quad+\delta(\chi)\left(1+q^{-1}\right)\left(\frac{q^{2 \lambda(t+1)}}{1-q^{2 \lambda}} \int_{\widetilde{K}_{H} \times \widetilde{K}_{H}} c(1, \lambda) \Psi_{S}(1)_{k_{1}, k_{2}} d k_{1} d k_{2}\right. \\
& \left.\quad+\frac{q^{-2 \lambda(t+1)}}{1-q^{-2 \lambda}} \int_{\widetilde{K}_{H} \times \widetilde{K}_{H}} c(w, \lambda) \Psi_{S}(1)_{k_{1}, k_{2}} d k_{1} d k_{2}\right),
\end{aligned}
$$

where $\delta(\chi)=1$ if $\left.\chi\right|_{\mathscr{O}_{F}^{\times}}=1$ and $\delta(\chi)=0$ if $\left.\chi\right|_{\mathscr{O}_{F}^{\times}} \neq 1$.

Proof. For $S \in \mathscr{B}_{P}(\chi), \Psi_{S} \in \mathscr{A}_{\chi}\left(M,\left(\tau_{\Gamma}\right)_{P \mid P}\right)$ and

$$
c(1, \lambda) \Psi_{S}, c(w, \lambda) \Psi_{S} \in \mathscr{A}_{\chi}\left(M,\left(\tau_{\Gamma}\right)_{P \mid \bar{P}}\right) .
$$

Therefore $\Psi=\Psi_{S}$ can be written as a sum of matrix coefficients of $\chi$. Thus

$$
\begin{aligned}
C^{P} E_{P}(m, \Psi, \lambda)_{k_{1}, k_{2}} & \\
& =c(1, \lambda) \Psi(m)_{k_{1}, k_{2}} e^{\lambda H_{M}(m)}+c(w, \lambda) \Psi(m)_{k_{1}, k_{2}} e^{-\lambda H_{M}(m)} \\
& =\chi(m)\left[(c(1, \lambda) \Psi)(1)_{k_{1}, k_{2}} e^{\lambda H_{M}(m)}+(c(w, \lambda) \Psi)(1)_{k_{1}, k_{2}} e^{-\lambda H_{M}(m)}\right]
\end{aligned}
$$

where $\chi(m) \in \mathbb{C}^{\times}$. Hence for $t \gg 0$,

$$
\begin{aligned}
& \int_{\widetilde{M}_{H}^{+}(F)} D_{P_{H}}(m) \operatorname{tr}\left(I_{P}\left(\chi_{\lambda}, k_{1} m k_{2}\right) S\right) e^{v\left(H_{M}(m)\right)}(1-u(m, t)) d m \\
& =\int_{\widetilde{M}_{H}^{+}(F)} D_{P_{H}}(m) \delta_{P}^{-\frac{1}{2}}(m)(c(1, \lambda) \Psi)(1)_{k_{1}, k_{2}} e^{(\lambda+v)\left(H_{M}(m)\right)} \chi(m)(1-u(m, t)) d m \\
& \quad+\int_{\widetilde{M}_{H}^{+}(F)} D_{P_{H}}(m) \delta_{P}^{-\frac{1}{2}}(m)(c(w, \lambda) \Psi)(1)_{k_{1}, k_{2}} e^{(-\lambda+v)\left(H_{M}(m)\right)} \chi(m)(1-u(m, t)) d m \\
& =\left(1+q^{-1}\right)(c(1, \lambda) \Psi)(1)_{k_{1}, k_{2}} \int_{\widetilde{M}_{H}^{+}(F)} e^{(\lambda+v)\left(H_{M}(m)\right)} \chi(m)(1-u(m, t)) d m \\
& \quad+\left(1+q^{-1}\right)(c(w, \lambda) \Psi)(1)_{k_{1}, k_{2}} \int_{\widetilde{M}_{H}^{+}(F)} e^{(-\lambda+v)\left(H_{M}(m)\right)} \chi(m)(1-u(m, t)) d m \\
& =\left(1+q^{-1}\right) \int_{\mathscr{O}_{F}^{\times}} \chi(\alpha) d^{\times} \alpha \sum_{n=t+1}^{\infty}\left[(c(1, \lambda) \Psi)(1)_{k_{1}, k_{2}} q^{2(\lambda+v) n}\right. \\
& \left.+(c(w, \lambda) \Psi)(1)_{k_{1}, k_{2}} q^{2(-\lambda+v) n}\right] .
\end{aligned}
$$


Clearly $\int_{\mathscr{O}_{F}^{\times}} \chi(\alpha) d^{\times} \alpha=0$ unless $\left.\chi\right|_{\mathscr{O}_{F}^{\times}}=1$. If $\left.\chi\right|_{\mathscr{O}_{F}^{\times}}=1$, the previous line equals

$$
\left(1+q^{-1}\right)\left(\frac{q^{2(\lambda+v)(t+1)}}{1-q^{2(\lambda+v)}} c(1, \lambda) \Psi(1)_{k_{1}, k_{2}}+\frac{q^{2(-\lambda+v)(t+1)}}{1-q^{2(-\lambda+v)}} c(w, \lambda) \Psi(1)_{k_{1}, k_{2}}\right) .
$$

Therefore for $t \gg 0$

$$
\begin{aligned}
& \int_{\widetilde{M}_{H}^{+}(F)}^{\sharp} D_{P_{H}}(m) \operatorname{tr}\left(I_{P}\left(\chi_{\lambda}, k_{1} m k_{2}\right) S\right)(1-u(m, t)) d m \\
& \quad=\delta(\chi)\left(1+q^{-1}\right)\left(\frac{q^{2 \lambda(t+1)}}{1-q^{2 \lambda}} c(1, \lambda) \Psi_{S}(1)_{k_{1}, k_{2}}+\frac{q^{-2 \lambda(t+1)}}{1-q^{-2 \lambda}} c(w, \lambda) \Psi_{S}(1)_{k_{1}, k_{2}}\right)
\end{aligned}
$$

and the proposition now follows.

Lemma 4.8. Let $\chi=\left(\chi, \chi^{-1}\right)$ where $\chi$ is a character of $E^{\times}$such that $\chi(\varpi)=1$. Then

(1) If $\left.\chi\right|_{F^{\times}} \neq 1$ and $\left.\chi\right|_{E^{1}} \neq 1$, then

$$
\int_{\widetilde{H}(F)}^{*} \operatorname{tr}\left(I_{P}\left(\chi_{\lambda}, h\right) S\right) d h=\int_{\widetilde{H}(F)} \operatorname{tr}\left(I_{P}\left(\chi_{\lambda}, h\right) u(h, t) d h=0 .\right.
$$

(2) If $\left.\chi\right|_{F^{\times}} \neq 1$ and $\left.\chi\right|_{E^{1}}=1$, then for $t \gg 0$,

$$
\int_{\widetilde{H}(F)}^{*} \operatorname{tr}\left(I_{P}\left(\chi_{\lambda}, h\right) S\right) d h=\int_{\widetilde{H}(F)} \operatorname{tr}\left(I_{P}\left(\chi_{\lambda}, h\right) S\right) u(h, t) d h .
$$

(3) If $\left.\chi\right|_{F^{\times}}=1$ and $\left.\chi\right|_{E^{1}} \neq 1$, then $\int_{\widetilde{H}}^{*} \operatorname{tr}\left(I_{P}\left(\chi_{\lambda}, h\right) S\right)$ dh is 0 whenever defined and

$$
\int_{\widetilde{K}_{H} \times \widetilde{K}_{H}} c(1, \lambda) \Psi_{S}(1)_{k_{1}, k_{2}} d k_{1} d k_{2}=\int_{\widetilde{K}_{H} \times \widetilde{K}_{H}} c(s, \lambda) \Psi_{S}(1)_{k_{1}, k_{2}} d k_{1} d k_{2}
$$

at $\lambda=0$.

(4) If $\left.\chi\right|_{F^{\times}}=1$ and $\left.\chi\right|_{E^{1}}=1$, then $\chi^{2}=1$. In this case $c(1, \lambda)$ and $c(s, \lambda)$ have a simple pole at $\lambda=0$ and so $\mu\left(\chi_{\lambda}\right)$ has a zero of order two at $\lambda=0$ and $\mu\left(\chi_{\lambda}\right) c(1, \lambda)=\mu\left(\chi_{\lambda}\right) c(s, \lambda)=0$ at $\lambda=0$.

In all cases,

$$
\mu\left(\chi_{\lambda}\right) \int_{\widetilde{H}(F)}^{*} \operatorname{tr}\left(I_{P}\left(\chi_{\lambda}, h\right) S\right) d h
$$

is holomorphic for all $\lambda \in i \mathbb{R}, S \in \mathscr{B}_{P}(\chi)$.

Proof. In this proof we follow the techniques of [Jacquet $\geq 2012$ ]. Case 2 is obvious from the above work. Case 1 is obvious from the above work and the $H$-invariance of $\int_{\widetilde{H}}^{*} \operatorname{tr}\left(I_{P}\left(\chi_{\lambda}, h\right) S\right) d h$ [Jacquet et al. 1999, Proposition 22]. 
The vanishing of the regularized period for $\lambda \neq 0$ in case 3 also follows from $H$-invariance. Then by the previous proposition we know that for $\lambda \neq 0$,

$$
\begin{aligned}
& \int_{\widetilde{H}(F)} \operatorname{tr}\left(I_{P}\left(\chi_{\lambda}, h\right) S\right) u(h, t) d h \\
&=-\left(1+q^{-1}\right)\left(\frac{q^{2 \lambda(t+1)}}{1-q^{2 \lambda}} \int_{\widetilde{K}_{H} \times \widetilde{K}_{H}} c(1, \lambda) \Psi_{S}(1)_{k_{1}, k_{2}} d k_{1} d k_{2}\right. \\
&\left.\quad+\frac{q^{-2 \lambda(t+1)}}{1-q^{-2 \lambda}} \int_{\widetilde{K}_{H} \times \widetilde{K}_{H}} c(w, \lambda) \Psi_{S}(1)_{k_{1}, k_{2}} d k_{1} d k_{2}\right) .
\end{aligned}
$$

Both sides are holomorphic and the left-hand side is also defined and holomorphic for $\lambda=0$. As

$$
\operatorname{Res}_{\lambda=0} \frac{q^{2 \lambda(t+1)}}{1-q^{2 \lambda}}=\frac{-1}{2 \log q} \quad \text { and } \quad \operatorname{Res}_{\lambda=0} \frac{q^{-2 \lambda(t+1)}}{1-q^{-2 \lambda}}=\frac{1}{2 \log q},
$$

we must have that

$$
\int_{\widetilde{K}_{H} \times \widetilde{K}_{H}} c(1,0) \Psi(1)_{k_{1}, k_{2}} d k_{1} d k_{2}=\int_{\widetilde{K}_{H} \times \widetilde{K}_{H}} c(w, 0) \Psi(1)_{k_{1}, k_{2}} d k_{1} d k_{2}
$$

In case 4 the poles and zeros are well-known and can also be seen by explicit computations of the intertwining operators. We have that

$$
\begin{aligned}
& \mu\left(\chi_{\lambda}\right) \int_{\widetilde{H}(F)} \operatorname{tr}\left(I_{P}\left(\chi_{\lambda}, h\right) S\right) u(h, t) d h \\
& =\mu\left(\chi_{\lambda}\right) \int_{\widetilde{H}(F)}^{*} \operatorname{tr}\left(I_{P}\left(\chi_{\lambda}, h\right) S\right) d h \\
& \quad-\left(1+q^{-1}\right) \mu\left(\chi_{\lambda}\right)\left(\frac{q^{2 \lambda(t+1)}}{1-q^{2 \lambda}} \int_{\widetilde{K}_{H} \times \widetilde{K}_{H}} c(1, \lambda) \Psi_{S}(1)_{k_{1}, k_{2}} d k_{1} d k_{2}\right. \\
& \left.\quad+\frac{q^{-2 \lambda(t+1)}}{1-q^{-2 \lambda}} \int_{\widetilde{K}_{H} \times \widetilde{K}_{H}} c(w, \lambda) \Psi_{S}(1)_{k_{1}, k_{2}} d k_{1} d k_{2}\right) .
\end{aligned}
$$

The left-hand side is 0 at $\lambda=0$ and the last two terms are holomorphic at $\lambda=0$ so the first term must be holomorphic at $\lambda=0$. 
Let

$$
\begin{aligned}
D_{\chi_{\lambda}}(f) & =\sum_{S \in \mathscr{B}_{P}(\chi)} P_{\chi_{\lambda}}\left(S_{\lambda}[f]\right) \overline{W_{\chi_{\lambda}}(S)}, \\
P_{\chi_{\lambda}}(S) & =\int_{\widetilde{H}(F)}^{*} \operatorname{tr}\left(I_{P}\left(\chi_{\lambda}, h\right) S\right) d h, \\
S_{\lambda}[f] & =I_{P}\left(\chi_{\lambda}, f_{2}\right) S I_{P}\left(\chi_{\lambda}, f_{1}^{\vee}\right), \\
\widetilde{D}_{\chi}(f) & =\left(1+q^{-1}\right) \mu\left(\chi_{0}\right) \sum_{S \in \mathscr{P}_{P}(\chi)} \overline{W_{\chi_{0}}(S)} \int_{\widetilde{K}_{H} \times \widetilde{K}_{H}} c(1,0) \psi_{S_{0}[f]}(1)_{k_{1}, k_{2}} d k_{1} d k_{2} .
\end{aligned}
$$

We now relate the distributions above to the truncated distributions from (4-2).

Lemma 4.9. Let $\chi=\left(\chi, \chi^{-1}\right)$ where $\chi$ is a character of $E^{\times}$such that $\chi(\varpi)=1$.

(1) If $\left.\chi\right|_{F^{\times}} \neq 1$ and $\left.\chi\right|_{E^{1}} \neq 1$, then

$$
\lim _{t \rightarrow \infty} \int_{0}^{\frac{\pi i}{\log q}} \mu\left(\chi_{\lambda}\right) D_{\chi_{\lambda}}^{t}(f) d \lambda=0 .
$$

(2) If $\left.\chi\right|_{F^{\times}} \neq 1$ and $\left.\chi\right|_{E^{1}}=1$, then

$$
\lim _{t \rightarrow \infty} \int_{0}^{\frac{\pi i}{\log q}} \mu\left(\chi_{\lambda}\right) D_{\chi_{\lambda}}^{t}(f) d \lambda=\int_{0}^{\frac{\pi i}{\log q}} \mu\left(\chi_{\lambda}\right) D_{\chi_{\lambda}}(f) d \lambda .
$$

(3) If $\left.\chi\right|_{F^{\times}}=1$ and $\left.\chi\right|_{E^{1}} \neq 1$, then

$$
\lim _{t \rightarrow \infty} \int_{0}^{\frac{\pi i}{\log q}} \mu\left(\chi_{\lambda}\right) D_{\chi_{\lambda}}^{t}(f) d \lambda=\widetilde{D}_{\chi}(f) .
$$

(4) If $\left.\chi\right|_{F^{\times}}=1$ and $\left.\chi\right|_{E^{1}}=1$, then

$$
\lim _{t \rightarrow \infty} \int_{0}^{\frac{\pi i}{\log q}} \mu\left(\chi_{\lambda}\right) D_{\chi_{\lambda}}^{t}(f) d \lambda=\int_{0}^{\frac{\pi i}{\log q}} \mu\left(\chi_{\lambda}\right) D_{\chi_{\lambda}}(f) d \lambda .
$$

Proof. First we note that

$$
\begin{aligned}
& \int_{0}^{\frac{\pi i}{\log q}} \mu\left(\chi_{\lambda}\right) D_{\chi_{\lambda}}^{t}(f) d \lambda=\int_{0}^{\frac{\pi i}{\log q}} \mu\left(\chi_{\lambda}\right) \sum_{S \in \mathscr{B}_{P}(\chi)} P_{I_{P}\left(\chi_{\lambda}\right)}^{t}\left(S_{\lambda}[f]\right) \overline{W_{\chi_{\lambda}}(S)} d \lambda \\
& =\sum_{S \in \mathscr{乃}_{P}(\chi)} \int_{0}^{\frac{\pi i}{\log q}}\left(\overline{\int_{N(F)} \operatorname{tr}\left(I_{P}\left(\chi_{\lambda}, n\right) S\right) \psi\left(n^{-1}\right) u(n, t) d n}\right) \\
& \times \mu\left(\chi_{\lambda}\right) \int_{\widetilde{H}(F)} \operatorname{tr}\left(I_{P}\left(\chi_{\lambda}, h\right) S_{\lambda}[f]\right) u(h, t) d h d \lambda .
\end{aligned}
$$


Cases 1 and 2 now follow directly from Lemma 4.8. For the remaining cases we note that by Proposition 4.7 for $t \gg 0$,

$$
\begin{aligned}
\int_{\widetilde{H}(F)} \operatorname{tr}\left(I_{P}\left(\chi_{\lambda}, h\right) S_{\lambda}[f]\right) u(h, t) d h & \\
= & \int_{\widetilde{H}(F)}^{*} \operatorname{tr}\left(I_{P}\left(\chi_{\lambda}, h\right) S_{\lambda}[f]\right) d h \\
& \quad+\delta(\chi) \frac{1+q^{-1}}{q^{\lambda}-q^{-\lambda}}\left(q^{2 \lambda\left(t+\frac{1}{2}\right)} \int_{\widetilde{K}_{H} \times \widetilde{K}_{H}} c(1, \lambda) \Psi_{S_{\lambda}[f]}(1)_{k_{1}, k_{2}} d k_{1} d k_{2}\right. \\
& \left.\quad-q^{-2 \lambda\left(t+\frac{1}{2}\right)} \int_{\widetilde{K}_{H} \times \widetilde{K}_{H}} c(w, \lambda) \Psi_{S_{\lambda}[f]}(1)_{k_{1}, k_{2}} d k_{1} d k_{2}\right) .
\end{aligned}
$$

In case 3 , by Lemma 4.8 the regularized period vanishes and we are left computing

$$
\begin{aligned}
\left(1+q^{-1}\right) \lim _{t \rightarrow \infty} \int_{0}^{\frac{\pi i}{\log q}} \mu\left(\chi_{\lambda}\right) \overline{W_{\chi_{\lambda}}(S)} \\
\quad\left(\frac{q^{2 \lambda(t+1 / 2)}}{q^{\lambda}-q^{-\lambda}} \int_{\widetilde{K}_{H} \times \widetilde{K}_{H}} c(1, \lambda) \Psi_{S_{\lambda}[f]}(1)_{k_{1}, k_{2}} d k_{1} d k_{2}\right. \\
\left.\quad-\frac{q^{-2 \lambda(t+1 / 2)}}{q^{\lambda}-q^{-\lambda}} \int_{\widetilde{K}_{H} \times \widetilde{K}_{H}} c(w, \lambda) \Psi_{S_{\lambda}[f]}(1)_{k_{1}, k_{2}} d k_{1} d k_{2}\right) d \lambda
\end{aligned}
$$

Let

$$
\begin{aligned}
f_{1}(\lambda)=\frac{1+q^{-1}}{2} \mu\left(\chi_{\lambda}\right) \overline{W_{\chi_{\lambda}}(S)}\left(\int_{\widetilde{K}_{H} \times \widetilde{K}_{H}} c(1, \lambda) \Psi_{S_{\lambda}[f]}(1)_{k_{1}, k_{2}} d k_{1} d k_{2}\right. \\
\left.-\int_{\widetilde{K}_{H} \times \widetilde{K}_{H}} c(w, \lambda) \Psi_{S_{\lambda}[f]}(1)_{k_{1}, k_{2}} d k_{1} d k_{2}\right), \\
f_{2}(\lambda)=\frac{1+q^{-1}}{2} \mu\left(\chi_{\lambda}\right) \overline{W_{\chi_{\lambda}}(S)}\left(\int_{\widetilde{K}_{H} \times \widetilde{K}_{H}} c(1, \lambda) \Psi_{S_{\lambda}[f]}(1)_{k_{1}, k_{2}} d k_{1} d k_{2}\right. \\
\left.+\int_{\widetilde{K}_{H} \times \widetilde{K}_{H}} c(w, \lambda) \Psi_{S_{\lambda}[f]}(1)_{k_{1}, k_{2}} d k_{1} d k_{2}\right) .
\end{aligned}
$$

Then (4-5) equals

$$
\begin{aligned}
& \lim _{t \rightarrow \infty} \int_{0}^{\frac{\pi i}{\log q}} \frac{f_{1}(\lambda)\left(q^{2 \lambda\left(t+\frac{1}{2}\right)}+q^{-2 \lambda\left(t+\frac{1}{2}\right)}\right)}{q^{\lambda}-q^{-\lambda}} \\
&+\lim _{t \rightarrow \infty} \int_{0}^{\frac{\pi i}{\log q}} \frac{f_{2}(\lambda)\left(q^{2 \lambda\left(t+\frac{1}{2}\right)}-q^{-2 \lambda\left(t+\frac{1}{2}\right)}\right)}{q^{\lambda}-q^{-\lambda}} d \lambda .
\end{aligned}
$$

By Lemma $4.8, f_{1}(0)=0$. Hence by Fourier analysis the first integral will vanish. The limit of the second integral will be $f_{2}(0)$, which, by the identity in case 3 of 
Lemma 4.8, equals

$$
\left(1+q^{-1}\right) \mu\left(\chi_{0}\right) \overline{W_{\chi_{0}}(S)} \int_{\widetilde{K}_{H} \times \widetilde{K}_{H}} c(1,0) \Psi_{S_{0}[f]}(1)_{k_{1}, k_{2}} d k_{1} d k_{2} .
$$

For case 4 by Lemma 4.8 when multiplied by $\mu\left(\chi_{\lambda}\right) \overline{W_{\chi_{\lambda}}(S)}, f_{1}(\lambda)$ and $f_{2}(\lambda)$ are holomorphic functions of $\lambda$ and vanish at $\lambda=0$, thus by similar analysis as above the last two terms vanish in the limit and we are left with the statement of the lemma.

4B1. Discrete series representations. Because the matrix coefficient of a supercuspidal representation $\sigma$ has compact support it is obvious that

$$
\lim _{t \rightarrow \infty} \int_{\widetilde{H}(F)} \operatorname{tr}(\sigma(h) S) u(h, t) d h=\int_{\widetilde{H}(F)} \operatorname{tr}(\sigma(h) S) d h .
$$

Now we will prove that this is also true for Steinberg representations.

Lemma 4.10. For $\sigma=S t(\chi), \chi^{2}=1$, the matrix coefficients are absolutely convergent over $\widetilde{H}(F)$. Thus the limit

$$
\lim _{t \rightarrow \infty} \int_{\widetilde{H}(F)} \operatorname{tr}(\sigma(h) S) u(h, t) d h
$$

exists and equals

$$
\int_{\widetilde{H}(F)} \operatorname{tr}(\sigma(h) S d h
$$

Proof. By [Borel and Wallach 1980, XI.4.3; Casselman 1995, 4.2.3], a matrix coefficient for $\sigma$ evaluated at $\left(\begin{array}{ll}a & 0 \\ 0 & b\end{array}\right)$ is equal to a matrix coefficient for the Jacquet functor $\sigma_{N}$, evaluated at the same value, for $\left|\frac{a}{b}\right|_{E}$ sufficiently small. The Jacquet functor of $\sigma$ is $\delta_{P}$. Thus outside some compact set, our original matrix coefficient will behave like $\delta_{P}$ on $M_{H}^{-}(F)$. When we integrate over $\widetilde{H}(F)$, using the $K_{H} M_{H}^{-}(F) K_{H}$ decomposition, we get a measure factor of $\delta_{P}^{-1 / 2}$. Thus outside a set of compact support our integral will look like $\int_{|a|<c}|a|_{F} d^{\times} a$ for some $c>0$.

Putting everything together we have proved the following.

Proposition 4.11. For any $f \in C_{c}^{\infty}(\widetilde{G}(F) \times \widetilde{G}(F))$,

$$
\begin{aligned}
\lim _{t \rightarrow \infty} \int_{\widetilde{H}(F)} \int_{N(F)} K_{f}(h, n) \psi(n) u(h, t) u(n, t) d n d h & \\
= & \sum_{\sigma \in \Pi_{2}(\widetilde{G}(F))} d(\sigma) D_{\sigma}(f)+\frac{1}{2} \sum_{\substack{\chi \in\left\{\Pi_{2}(\widetilde{M}(F))\right\} \\
\chi^{2} \neq 1,\left.\chi\right|_{F}=1}} \widetilde{D}_{\chi}(f) \\
& +\frac{1}{2} \sum_{\substack{\left.\chi \in\left\{\Pi_{2}(\widetilde{M}(F))\right\} \\
\chi\right|_{E^{1}}=1}} d(\chi) \int_{0}^{\frac{\pi i}{\log q}} \mu\left(\chi_{\lambda}\right) D_{\chi_{\lambda}}(f) d \lambda,
\end{aligned}
$$


where

$$
\begin{aligned}
D_{\chi_{\lambda}}(f)= & \sum_{S \in \mathscr{B}_{P}(\chi)} P_{\chi_{\lambda}}\left(S_{\lambda}[f]\right) \overline{W_{\chi_{\lambda}}(S)}, \\
P_{\chi_{\lambda}}(S)= & \int_{\widetilde{H}(F)}^{*} \operatorname{tr}\left(I_{P}\left(\chi_{\lambda}, h\right) S\right) d h, \\
\widetilde{D}_{\chi}(f)= & \left(1+q^{-1}\right) \mu\left(\chi_{0}\right) \sum_{S \in \mathscr{B}_{P}(\chi)} \overline{W_{\chi_{0}}(S)} \int_{\widetilde{K}_{H} \times \widetilde{K}_{H}} c(1,0) \psi_{S_{0}[f]}(1)_{k_{1}, k_{2}} d k_{1} d k_{2}, \\
D_{\sigma}(f)= & \sum_{S \in \mathscr{B}(\sigma)} P_{\sigma}\left(\sigma\left(f_{2}\right) S \sigma\left(f_{1}^{\vee}\right)\right) \overline{W_{\sigma}(S)}, \\
P_{\sigma}(S)= & \int_{\widetilde{H}(F)} \operatorname{tr}(\sigma(h) S) d h .
\end{aligned}
$$

This proposition combined with Proposition 4.3 proves Theorem 1.4.

\section{Comparison of local trace formulas and applications}

We now combine the results of the previous two sections to compare the two trace formulas. Let $\omega_{E / F}$ be the quadratic character of $F^{\times}$associated to $E / F$ and let $\omega$ denote its trivial extension to $E^{\times}$.

Definition 5.1. We say that $f^{\prime} \in C_{c}^{\infty}\left(\widetilde{G}^{\prime}(F)\right)$ and $f \in C_{c}^{\infty}(\widetilde{G}(F))$ are matching functions if $O^{\prime}\left(f^{\prime}, \psi^{\prime}, a\right)=\omega(a) O(f, \psi, a)$ for all $a \in E^{\times}$.

By work of Ye [1989] and Flicker [1991, Proposition 3], we know that for any $f^{\prime} \in C_{c}^{\infty}\left(\widetilde{G}^{\prime}(F)\right)$ there exists a matching $f \in C_{c}^{\infty}(\widetilde{G}(F))$ and vice versa. In fact, by the Fundamental Lemma, for $f^{\prime}$ spherical, we know that $f$ is the corresponding function from the base change map between their Hecke algebras. Thus by the geometric expansion of the trace formulas in Propositions 3.4 and 4.3 we have the following statement.

Proposition 5.2. For $f_{i}^{\prime} \in C_{c}^{\infty}\left(\widetilde{G}^{\prime}(F)\right)$ and $f_{i} \in C_{c}^{\infty}(\widetilde{G}(F))$ matching functions for $i=1,2$,

$$
\begin{array}{r}
\lim _{t \rightarrow \infty} \int_{N^{\prime}(F)} \int_{N^{\prime}(F)} K_{f_{1}^{\prime} \otimes f_{2}^{\prime}}\left(n_{1}, n_{2}\right) \psi^{\prime}\left(n_{1}^{-1} n_{2}\right) u\left(n_{1}, t\right) u\left(n_{2}, t\right) d n_{1} d n_{2} \\
=\lim _{t \rightarrow \infty} \int_{\widetilde{H}(F)} \int_{N(F)} K_{f_{1} \otimes f_{2}}(h, n) \psi(n) u(h, t) u(n, t) d n d h .
\end{array}
$$

Now we use the equality of the trace formulas to compare the spectral expansions. By Propositions 3.6, 4.11 and 5.2 we have the following result. 
Theorem 5.3. For $f_{i}$ and $f_{i}^{\prime}$ matching functions for $i=1,2$,

$$
\begin{gathered}
\sum_{\sigma^{\prime} \in \Pi_{2}\left(\widetilde{G}^{\prime}(F)\right)} d\left(\sigma^{\prime}\right) D_{\sigma^{\prime}}^{\prime}\left(f_{1}^{\prime} \otimes f_{2}^{\prime}\right)+\frac{1}{2} \sum_{\substack{\chi^{\prime} \in\left\{\Pi_{2}\left(\widetilde{M}^{\prime}(F)\right)\right\} \\
\sigma \in \Pi_{2}(\widetilde{G}(F))}} d\left(\chi^{\prime}\right) \int_{0}^{\frac{\pi i}{\log q}} \mu\left(\chi_{\lambda}^{\prime}\right) D_{\chi_{\lambda}^{\prime}}^{\prime}\left(f_{1}^{\prime} \otimes f_{2}^{\prime}\right) d \lambda \\
=\sum_{\substack{\chi \in\left\{\Pi_{2}(\widetilde{M}(F))\right\} \\
\chi^{2} \neq 1,\left.\chi\right|_{F}=1}} \widetilde{D}_{\chi}\left(f_{1} \otimes f_{2}\right) \\
+\frac{1}{2} \sum_{\substack{\left.\chi \in\left\{\Pi_{2}(\widetilde{M}(F))\right\} \\
\chi\right|_{E^{1}}=1}} d(\chi) \int_{0}^{\frac{\pi i}{\log q}} \mu\left(\chi_{\lambda}\right) D_{\chi_{\lambda}}\left(f_{1} \otimes f_{2}\right) d \lambda .
\end{gathered}
$$

The unstable base change map associated to $\omega$ lifts principal series representations of $\widetilde{G}^{\prime}$ to principal series representations $I_{P}(\chi)$ of $\widetilde{G}$ such that $\left.\chi\right|_{E^{1}}=1$. It also lifts certain square integrable representations of $\widetilde{G}^{\prime}$ to the principal series representations of $\widetilde{G}$ defined by $I_{P}(\chi \omega)$ such that $\chi^{2} \neq 1,\left.\chi\right|_{F^{\times}}=1$. It lifts the remaining square integrable representations of $\widetilde{G}^{\prime}$ to square integral representations of $\widetilde{G}$ [Rogawski 1990; Flicker 1982]. Thus we could rephrase the right-hand side of Theorem 5.3 in terms of summing over the representations of $\widetilde{G}$ that are the unstable base change lifts of representations of $\widetilde{G}^{\prime}$. The extra discrete term $\widetilde{W}_{\chi}(f)$ corresponds exactly to the representations that lift from the discrete series of $\widetilde{G}^{\prime}$ to the principal series of $\widetilde{G}$.

We also note that the only representations that appear on the right-hand side of Theorem 5.3 are those $\sigma$ or $I_{P}\left(\chi_{\lambda}\right)$ for which there is a matrix coefficient such that the regularized integral over $H$ is nonzero. This gives us a more explicit description of the nonvanishing $H$ invariant linear functional that characterizes the image of the unstable base change map.

We would like to relate our distributions to the local factors in the Bessel and relative Bessel distributions. Recall from the introduction that Jacquet's global relative trace formula tells us that for $f^{\prime}$ on $U\left(2, \mathbb{A}_{F}\right)$ and $f$ on $\operatorname{GL}\left(2, \mathbb{A}_{E}\right)$ matching functions, if a cuspidal representation $\pi^{\prime}$ of $U\left(2, \mathbb{A}_{F}\right)$ maps to $\pi$ of $\operatorname{GL}\left(2, \mathbb{A}_{E}\right)$ under unstable base change, then

$$
B_{\pi^{\prime}}^{\prime}\left(f^{\prime}\right)=B_{\pi}(f)
$$


where

$$
\begin{aligned}
B_{\pi^{\prime}}^{\prime}\left(f^{\prime}\right) & =\sum_{\phi^{\prime} \in \text { o.n.b. }\left(V_{\pi^{\prime}}\right)} W^{\prime}\left(\pi^{\prime}\left(f^{\prime}\right) \phi^{\prime}\right) \overline{W^{\prime}\left(\phi^{\prime}\right)}, \\
B_{\pi}(f) & =\sum_{\phi \in \text { o.n.b. }\left(V_{\pi}\right)} P(\pi(f) \phi) \overline{W(\phi)}, \\
W^{\prime}\left(\phi^{\prime}\right) & =\int_{N^{\prime}(F) \backslash N^{\prime}\left(\mathbb{A}_{F}\right)} \phi^{\prime}(n) \overline{\psi^{\prime}(n)} d n, \\
W(\phi) & =\int_{N(E) \backslash N\left(\mathbb{A}_{E}\right)} \phi(n) \overline{\psi(n)} d n, \\
P(\phi) & =\int_{\mathrm{GL}(2, F) Z\left(\mathbb{A}_{F}\right) \backslash \mathrm{GL}\left(2, \mathbb{A}_{F}\right)} \phi(h) d h \neq 0 .
\end{aligned}
$$

While $B_{\pi^{\prime}}^{\prime}\left(f^{\prime}\right)$ and $B_{\pi}(f)$ factor into local Bessel distributions $B_{\pi_{v}^{\prime}}^{\prime}\left(f_{v}^{\prime}\right)$ and $B_{\pi_{v}}\left(f_{v}\right)$, it is not clear how to normalize the local Bessel distributions. We can rewrite our local distributions as a product of two local Bessel (or local relative Bessel) distributions:

Lemma 5.4. (1) For $\sigma^{\prime}$ an irreducible supercuspidal representation of $\widetilde{G}^{\prime}(F)$, there exists a local Bessel distribution $B_{\sigma^{\prime}}^{\prime}$, unique up to a constant of absolute value 1 , such that

$$
D_{\sigma^{\prime}}^{\prime}\left(f_{1}^{\prime} \otimes f_{2}^{\prime}\right)=B_{\sigma^{\prime}}^{\prime}\left(f_{2}^{\prime}\right) B_{\sigma^{* *}}^{\prime}\left(f_{1}^{\prime}\right)
$$

(2) For $\sigma$ an irreducible supercuspidal representation of $\widetilde{G}(F)$, there exists a local relative Bessel distribution $B_{\sigma}$, unique up to a constant of absolute value 1 , such that

$$
D_{\sigma}\left(f_{1} \otimes f_{2}\right)=B_{\sigma}\left(f_{2}\right) B_{\sigma^{*}}\left(f_{1}\right) .
$$

Proof. We recall that

$$
\begin{aligned}
& D_{\sigma^{\prime}}^{\prime}\left(f^{\prime}\right)=\sum_{S^{\prime} \in \mathscr{P}\left(\sigma^{\prime}\right)} \int_{N^{\prime}(F)} \operatorname{tr}\left(\sigma^{\prime}\left(n_{1}\right) \sigma^{\prime}\left(f_{2}^{\prime}\right) S^{\prime} \sigma^{\prime *}\left(f_{1}^{\prime}\right)\right) \psi^{\prime}\left(n_{1}\right)^{-1} d n_{1} \\
& \overline{\int_{N^{\prime}(F)} \operatorname{tr}\left(\sigma^{\prime}\left(n_{2}\right) S^{\prime}\right) \psi^{\prime}\left(n_{2}\right)^{-1} d n_{2}} .
\end{aligned}
$$

Let $V=V_{\sigma^{\prime}}$. As $S^{\prime}$ is an endomorphism on $V$ there exist $v \in V, v^{*} \in V^{*}$ such that $S^{\prime}=v \otimes v^{*}$. Then the linear functional on $V \otimes V^{*}$ that acts by

$$
v \otimes v^{*} \mapsto \int_{N^{\prime}(F)} \operatorname{tr}\left(\sigma^{\prime}(n) v \otimes v^{*}\right) \psi^{\prime}(n)^{-1} d n
$$


transforms under $n$ on $v$ and $v^{*}$ by $\psi^{\prime}$. Thus it is a Whittaker functional on $V \otimes V^{*}$. By the uniqueness of Whittaker models,

$$
\int_{N^{\prime}(F)} \operatorname{tr}\left(\sigma^{\prime}(n) S^{\prime}\right) \psi^{\prime}(n)^{-1} d n=W^{\prime}(v) W^{\prime}\left(v^{*}\right) .
$$

Thus

$$
\begin{aligned}
D_{\sigma^{\prime}}^{\prime}\left(f^{\prime}\right) & =\sum_{v \otimes v^{*}} W^{\prime}\left(\sigma^{\prime}\left(f_{2}^{\prime}\right) v\right) \overline{W^{\prime}(v)} W^{\prime}\left(\sigma^{*}\left(f_{1}^{\prime}\right) v^{*}\right) \overline{W^{\prime}\left(v^{*}\right)} \\
& =B_{\sigma^{\prime}}^{\prime}\left(f_{2}^{\prime}\right) B_{\sigma^{\prime *}}^{\prime}\left(f_{1}^{\prime}\right) .
\end{aligned}
$$

We note that if we change $B_{\sigma^{\prime}}^{\prime}$ by a constant $c$, then $B_{\sigma^{*}}^{\prime}$ will change by $\bar{c}$.

The proof for the local relative Bessel distributions is similar, using the uniqueness of the $H$-invariant linear functional [Hakim 1991; Flicker 1991, Proposition 11].

We can also describe matching functions by an equality of all the Bessel distributions.

Lemma 5.5 (density). (1) If $f_{1}^{\prime} \in C_{c}^{\infty}\left(\widetilde{G}^{\prime}(F)\right)$ is such that $D_{\sigma^{\prime}}^{\prime}\left(f_{1}^{\prime} \otimes f_{2}^{\prime}\right)=0$ for all irreducible tempered representations $\sigma^{\prime}$ of $\widetilde{G}^{\prime}(F)$ and all $f_{2}^{\prime}$, then $O^{\prime}\left(f_{1}^{\prime}, \psi^{\prime-1}, a\right)=0$ for all $a \in E^{\times}$.

(2) If $f_{1} \in C_{c}^{\infty}(\widetilde{G}(F))$ is such that $D_{\sigma}\left(f_{1} \otimes f_{2}\right)=0$ and $\widetilde{D}_{\sigma}\left(f_{1} \otimes f_{2}\right)=0$ for all irreducible tempered representations $\sigma$ of $\widetilde{G}(F)$ and $f_{2}$, then $O\left(f_{1}, \psi^{-1}, a\right)=0$ for all $a \in E^{\times}$.

Proof. If $D_{\sigma^{\prime}}^{\prime}\left(f_{1}^{\prime} \otimes f_{2}^{\prime}\right)=0$ for all $\sigma^{\prime}$, then by Theorem 1.3,

$$
\int_{a \in E^{\times} / E^{1}}|a|_{E} O^{\prime}\left(f_{1}^{\prime}, \psi^{\prime-1}, a\right) O^{\prime}\left(f_{2}^{\prime}, \psi^{\prime}, a\right) d^{\times} a=0
$$

for all $f_{2}^{\prime} \in C_{c}^{\infty}\left(\widetilde{G}^{\prime}(F)\right)$. As $O^{\prime}\left(f_{1}^{\prime}, \psi^{-1}, a\right)$ is a locally constant function of $a$ there exists some open compact $U$ such that $O^{\prime}\left(f_{1}^{\prime}, \psi^{\prime-1}, a\right)$ is biinvariant under it. Then by choosing $f_{2}^{\prime}$ such that $O^{\prime}\left(f_{2}^{\prime}, \psi^{\prime-1}, a\right)$ has support contained in $U$ we see that $O^{\prime}\left(f_{1}^{\prime}, \psi^{\prime-1}, a\right)=0$. The second case follows from the first one.

Combining Theorem 5.3 with Lemma 5.4 and the global relative trace formula, we have the following result:

Corollary 5.6. If $\sigma$ is the supercuspidal representation of $\widetilde{G}(F)$ that is the unstable base change lift of the supercuspidal representation $\sigma^{\prime}$ on $\widetilde{G}^{\prime}(F)$, and $f_{i}^{\prime}$ and $f_{i}$ are matching functions for $i=1,2$, then

$$
d\left(\sigma^{\prime}\right) D_{\sigma^{\prime}}\left(f_{1}^{\prime} \otimes f_{2}^{\prime}\right)=d(\sigma) D_{\sigma}\left(f_{1} \otimes f_{2}\right) .
$$

Proof. From the global comparison of relative trace formulas [Flicker 1991; Lapid 2006; Ye 1989] and a standard globalization argument we know there exists a constant $c_{\sigma}$ such that $B_{\sigma^{\prime}}^{\prime}\left(f_{i}^{\prime}\right)=c_{\sigma} B_{\sigma}\left(f_{i}\right)$ for all matching $f_{i}, f_{i}^{\prime}$. Take $f_{1}^{\prime}$ and $f_{2}$ to 
be matrix coefficients of $\sigma^{\prime}$ and $\sigma$ such that $B_{\sigma^{\prime}}^{\prime}\left(f_{1}^{\prime}\right) \neq 0$ and $B_{\sigma}\left(f_{2}\right) \neq 0$. Take $f_{2}^{\prime}$ a matching function to $f_{2}$ and $f_{1}$ a matching function to $f_{1}^{\prime}$. Then by Theorem 5.3,

$$
d\left(\sigma^{\prime}\right) D_{\sigma^{\prime}}^{\prime}\left(f^{\prime}\right)=d(\sigma) D_{\sigma}(f) .
$$

In addition to the spectral comparison, these local trace formulas also have applications on the geometric side. If we define the inner product of two functions $g_{1}, g_{2}$ on $E^{\times} / E^{1}$ by

$$
\left\langle g_{1}, g_{2}\right\rangle=\int_{a \in E^{\times} / E^{1}} g_{1}(a) g_{2}(a)|a|_{E} d^{\times} a,
$$

then:

Corollary 5.7 (orthogonality relations). For $f_{1}$ and $f_{2}$ matrix coefficients of the supercuspidal representations $\sigma_{1}$ and $\sigma_{2}$ of $\widetilde{G}(F)$,

$$
\left\langle O\left(f_{1}, \psi, \cdot\right), O\left(f_{2}, \psi^{-1}, \cdot\right)\right\rangle \neq 0 \Longleftrightarrow \sigma_{1} \sim \sigma_{2} .
$$

For $f_{1}^{\prime}$ and $f_{2}^{\prime}$ matrix coefficients of the supercuspidal representations $\sigma_{1}^{\prime}$ and $\sigma_{2}^{\prime}$ of $\widetilde{G}^{\prime}(F)$,

$$
\left\langle O^{\prime}\left(f_{1}^{\prime}, \psi^{\prime}, \cdot\right), O^{\prime}\left(f_{2}^{\prime}, \psi^{\prime-1}, \cdot\right)\right\rangle \neq 0 \Longleftrightarrow \sigma_{1}^{\prime} \sim \sigma_{2}^{\prime} .
$$

Proof. This follows directly from the local Kuznetsov and local relative trace formulas.

\section{Acknowledgments}

The author thanks Erez Lapid for his helpful suggestions in an early stage of this project and Omer Offen for his useful comments. Finally, the author thanks the referee for helpful comments.

\section{References}

[Arthur 1989] J. Arthur, "Towards a local trace formula”, pp. 1-23 in Algebraic analysis, geometry, and number theory (Baltimore, MD, 1988), edited by J.-I. Igusa, Johns Hopkins Univ. Press, Baltimore, MD, 1989. MR 98h:22020 Zbl 0765.22010

[Arthur 1991] J. Arthur, "A local trace formula”, Inst. Hautes Études Sci. Publ. Math. 73 (1991), 5-96. MR 92f:22029 Zbl 0741.22013

[Borel and Wallach 1980] A. Borel and N. R. Wallach, Continuous cohomology, discrete subgroups, and representations of reductive groups, Annals of Mathematics Studies 94, Princeton University Press, 1980. MR 83c:22018 Zbl 0443.22010

[Casselman 1995] B. Casselman, "Introduction to the theory of admissible representations of $p$ adic reductive groups", unpublished notes, 1995, Available at www.math.ubc.ca/ cass/research/pdf/ p-adic-book.pdf.

[Flicker 1982] Y. Z. Flicker, "Stable and labile base change for U(2)", Duke Math. J. 49:3 (1982), 691-729. MR 84i:22016 Zbl 0502.12013 
[Flicker 1991] Y. Z. Flicker, "On distinguished representations", J. Reine Angew. Math. 418 (1991), 139-172. MR 92i:22019 Zbl 0725.11026

[Hakim 1991] J. Hakim, "Distinguished p-adic representations", Duke Math. J. 62:1 (1991), 1-22. MR 92c:22037 Zbl 0724.22016

[Harish-Chandra 1976] Harish-Chandra, "Harmonic analysis on real reductive groups, III: The MaassSelberg relations and the Plancherel formula", Ann. of Math. (2) 104:1 (1976), 117-201. MR 55 \#12875 Zbl 0331.22007

[Harish-Chandra 1984] Harish-Chandra, "The plancherel formula for reductive $p$-adic groups", pp. 353-367 in Collected papers Vol. IV, edited by V. S. Varadarajan, Springer, New York, 1984. MR 85e:01061d

[Jacquet 2005] H. Jacquet, "A guide to the relative trace formula", pp. 257-272 in Automorphic representations, L-functions and applications: Progress and prospects, edited by J. W. Cogdell et al., Ohio State Univ. Math. Res. Inst. Publ. 11, de Gruyter, Berlin, 2005. MR 2006g:11100 Zbl 1106.11016

[Jacquet $\geq 2012$ ] H. Jacquet, "Truncation for the Fourier trace formula: the GL(2) case", unpublished notes.

[Jacquet and Lai 1985] H. Jacquet and K. F. Lai, "A relative trace formula", Compositio Math. 54:2 (1985), 243-310. MR 86j:11059 Zbl 0587.12006

[Jacquet et al. 1999] H. Jacquet, E. Lapid, and J. Rogawski, "Periods of automorphic forms", J. Amer. Math. Soc. 12:1 (1999), 173-240. MR 99c:11056 Zbl 1012.11044

[Lapid 2006] E. M. Lapid, "On the fine spectral expansion of Jacquet's relative trace formula", J. Inst. Math. Jussieu 5:2 (2006), 263-308. MR 2007d:11059 Zbl 1195.11071

[Rogawski 1990] J. D. Rogawski, Automorphic representations of unitary groups in three variables, Annals of Mathematics Studies 123, Princeton University Press, 1990. MR 91k:22037 Zbl 0724.11031

[Silberger 1996] A. J. Silberger, "Harish-Chandra's Plancherel theorem for p-adic groups", Trans. Amer. Math. Soc. 348:11 (1996), 4673-4686. MR 99c:22026 Zbl 0869.22007

[Springer 1985] T. A. Springer, "Some results on algebraic groups with involutions", pp. 525-543 in Algebraic groups and related topics (Kyoto/Nagoya, 1983), edited by R. Hotta, Adv. Stud. Pure Math. 6, North-Holland, Amsterdam, 1985. MR 86m:20050 Zbl 0628.20036

[Waldspurger 2003] J.-L. Waldspurger, "La formule de Plancherel pour les groupes $p$-adiques (d'après Harish-Chandra)", J. Inst. Math. Jussieu 2:2 (2003), 235-333. MR 2004d:22009 Zbl 1029.22016

[Ye 1989] Y. Ye, "Kloosterman integrals and base change for GL(2)", J. Reine Angew. Math. 400 (1989), 57-121. MR 90i:11134 Zbl 0665.10020

Received July 19, 2012. Revised October 9, 2012.

\section{BROOKE FEIGON}

DEPARTMENT OF MATHEMATICS

The City College of New York, CUNY

NAC $8 / 133$

NEW YORK, NY 10031

UNITED STATES

bfeigon@ccny.cuny.edu 


\title{
PACIFIC JOURNAL OF MATHEMATICS
}

\author{
http://pacificmath.org
}

Founded in 1951 by E. F. Beckenbach (1906-1982) and F. Wolf (1904-1989)

\section{EDITORS}

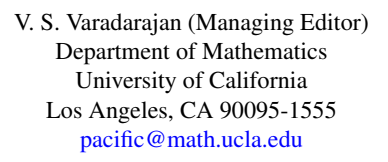

Don Blasius

Department of Mathematics University of California

Los Angeles, CA 90095-1555

blasius@math.ucla.edu

Robert Finn

Department of Mathematics

Stanford University

Stanford, CA 94305-2125

finn@math.stanford.edu

Alexander Merkurjev

Department of Mathematics

University of California

Los Angeles, CA 90095-1555

merkurev@math.ucla.edu

\author{
Vyjayanthi Chari \\ Department of Mathematics \\ University of California \\ Riverside, CA 92521-0135 \\ chari@math.ucr.edu \\ Kefeng Liu \\ Department of Mathematics \\ University of California \\ Los Angeles, CA 90095-1555 \\ liu@math.ucla.edu \\ Sorin Popa \\ Department of Mathematics \\ University of California \\ Los Angeles, CA 90095-1555 \\ popa@math.ucla.edu \\ Paul Yang \\ Department of Mathematics \\ Princeton University \\ Princeton NJ 08544-1000 \\ yang@math.princeton.edu
}

\section{PRODUCTION}

Silvio Levy, Scientific Editor, pacific@math.berkeley.edu

\section{SUPPORTING INSTITUTIONS}

ACADEMIA SINICA, TAIPEI

CALIFORNIA INST. OF TECHNOLOGY

INST. DE MATEMÁTICA PURA E APLICADA

KEIO UNIVERSITY

MATH. SCIENCES RESEARCH INSTITUTE

NEW MEXICO STATE UNIV.

OREGON STATE UNIV.

\author{
STANFORD UNIVERSITY \\ UNIV. OF BRITISH COLUMBIA \\ UNIV. OF CALIFORNIA, BERKELEY \\ UNIV. OF CALIFORNIA, DAVIS \\ UNIV. OF CALIFORNIA, LOS ANGELES \\ UNIV. OF CALIFORNIA, RIVERSIDE \\ UNIV. OF CALIFORNIA, SAN DIEGO \\ UNIV. OF CALIF., SANTA BARBARA
}

\author{
Daryl Cooper \\ Department of Mathematics \\ University of California \\ Santa Barbara, CA 93106-3080 \\ cooper@math.ucsb.edu \\ Jiang-Hua Lu \\ Department of Mathematics \\ The University of Hong Kong \\ Pokfulam Rd., Hong Kong \\ jhlu@maths.hku.hk
}

\section{Jie Qing}

Department of Mathematics

University of California

Santa Cruz, CA 95064

qing@cats.ucsc.edu

These supporting institutions contribute to the cost of publication of this Journal, but they are not owners or publishers and have no responsibility for its contents or policies.

See inside back cover or pacificmath.org for submission instructions.

The subscription price for 2012 is US \$420/year for the electronic version, and \$485/year for print and electronic.

Subscriptions, requests for back issues from the last three years and changes of subscribers address should be sent to Pacific Journal of Mathematics, P.O. Box 4163, Berkeley, CA 94704-0163, U.S.A. Prior back issues are obtainable from Periodicals Service Company, 11 Main Street, Germantown, NY 12526-5635. The Pacific Journal of Mathematics is indexed by Mathematical Reviews, Zentralblatt MATH, PASCAL CNRS Index, Referativnyi Zhurnal, Current Mathematical Publications and the Science Citation Index.

The Pacific Journal of Mathematics (ISSN 0030-8730) at the University of California, c/o Department of Mathematics, 969 Evans Hall, Berkeley, CA 94720-3840, is published monthly except July and August. Periodical rate postage paid at Berkeley, CA 94704, and additional mailing offices. POSTMASTER: send address changes to Pacific Journal of Mathematics, P.O. Box 4163, Berkeley, CA 94704-0163.

PJM peer review and production are managed by EditFlow ${ }^{\circledR}$ from Mathematical Sciences Publishers.

\section{PUBLISHED BY}

\section{mathematical sciences publishers}

http://msp.org/

A NON-PROFIT CORPORATION

Typeset in LATEX 


\section{PACIFIC JOURNAL OF MATHEMATICS}

Volume $260 \quad$ No. $2 \quad$ December 2012

\section{Special issue \\ devoted to the memory of Jonathan Rogawski}

In memoriam: Jonathan Rogawski

257

DON BLASIUS, DINAKAR RAMAKRISHNAN and V. S. VARADARAJAN

$p$-adic Rankin $L$-series and rational points on CM elliptic curves

261

Massimo Bertolini, Henri DARMON and KARTIK PRASANNA

The syntomic regulator for $K_{4}$ of curves

AMNON BESSER and ROB DE JEU

Unique functionals and representations of Hecke algebras

381

BENJAMIN BRUBAKER, DANIEL BUMP and SOLOMON FRIEDBERG

A relative trace formula for PGL(2) in the local setting

395

BROOKE FEIGON

On the degrees of matrix coefficients of intertwining operators

433

TOBIAS FINIS, EREZ LAPID and WERNER MÜLlER

Comparison of compact induction with parabolic induction

457

Guy HENNIART and MARIE-FranCE Vigneras

The functional equation and beyond endoscopy

497

P. EDWARD HERMAN

A correction to Conducteur des Représentations du groupe linéaire

HERVÉ JACQUET

Modular $L$-values of cubic level

ANDREW KNIGHTLY and CHARLES LI

On occult period maps

STEPHEN KUDLA and MiCHAEL RAPOPORT

A prologue to "Functoriality and reciprocity", part I

ROBERT LANGLANDS

Truncation of Eisenstein series

EREZ LAPID and KeITH OUELLETTE

Some comments on Weyl's complete reducibility theorem

JONATHAN ROGAWSKI and V. S. VARADARAJAN

On equality of arithmetic and analytic factors through local Langlands correspondence

FREYDOON SHAHIDI 